\title{
Combining 4- and 3-direction Subdivision
}

\author{
Jörg Peters \\ and \\ Le-Jeng Shiue \\ University of Florida
}

4-3 direction subdivision combines quad and triangle meshes. On quad submeshes it applies a 4-direction alternative to Catmull-Clark subdivision and on triangle submeshes a modification of Loop's scheme. Remarkably, 4-3 surfaces can be proven to be $C^{1}$ and have bounded curvature everywhere. In regular mesh regions, they are $C^{2}$ and correspond to two closely-related boxsplines of degree four. The box-spline in quad regions has a smaller stencil than Catmull-Clark and defines the unique scheme with a $3 \times 3$ stencil that can model constant features without ripples both aligned with the quad grid and diagonal to it. From a theoretical point of view, 4-3 subdivision near extraordinary points is remarkable in that the eigenstructure of the local subdivision matrix is easy to determine and a complete analysis is possible. Without tweaking the rules artificially to force a specific spectrum, the leading eigenvalues ordered by modulus of all local subdivision matrices are $1, \frac{1}{2}, \frac{1}{2}, \frac{1}{4}$ where the multiplicity of the eigenvalue $\frac{1}{4}$ depends on the valence of the extraordinary point and the number of quads surrounding it. This implies equal refinement of the mesh, regardless of the number of neighbors of a mesh node.

Categories and Subject Descriptors: I.3.5 [Computer Graphics]: surface representation, splines; I.3.6 [Computer Graphics]: graphics data structures

General Terms: Algorithms

Additional Key Words and Phrases: Subdivision, Geometric Modeling, CAD, Curves \& Surfaces

\section{INTRODUCTION}

Tensor-product surface constructions are known to exhibit aliasing and oscillation when geometric features are not aligned with the parameter lines (see, for example, the surfaces labeled CC in Figure 1). In fact, all control-mesh based schemes, whether spline-based or subdivision-based, are sensitive to the alignment of features with the mesh lines due to the shape (of the support) of the, finitely many, associated basis functions. However, for tensor-product schemes, the lack of constancy in the direction diagonal to the tensor-grid, is particularly noticeable: by raising a sequence of control points along the diagonal, an artist, focusing on geometry and unaware of underlying basis functions, may well expect to see a constant ridge rather than the sequence of camel humps arising from tensor-product constructions (Figure 1). In practice, such lack of directional monotonicity forces design

Author's address: Jörg Peters, Dept C.I.S.E., CSE Bldg, University of Florida, Gainesville, FL 32611-6120, email:jorg@ cise.ufl.edu; Le-Jeng Shiue, Dept C.I.S.E., CSE Bldg, University of Florida, Gainesville, FL 326116120, email:sle-jeng@cise.ufl.edu;

Permission to make digital/hard copy of all or part of this material without fee for personal or classroom use provided that the copies are not made or distributed for profit or commercial advantage, the ACM copyright/server notice, the title of the publication, and its date appear, and notice is given that copying is by permission of the ACM, Inc. To copy otherwise, to republish, to post on servers, or to redistribute to lists requires prior specific permission and/or a fee.

(c) 20YY ACM 0730-0301/20YY/0100-0001 $\$ 5.00$

ACM Transactions on Graphics, Vol. V, No. N, Month 20YY, Pages 1-25. 

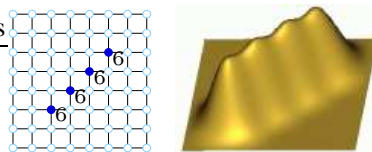

$\mathrm{CC}$

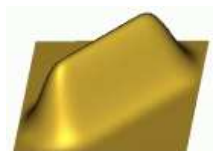

4-3
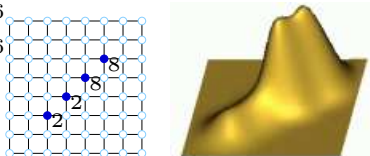

CC

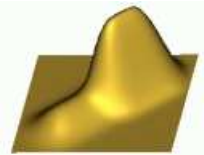

4-3

Fig. 1. Both the input control nets are planar except for the raised interior diagonal nodes whose values are displayed. Catmull-Clark (CC) subdivision exhibits ripples, 4-3 subdivision does not.

artists to lay out meshes carefully, avoiding complex joints altogether or adding features as displacement maps. To help the designer, a number of modifications of the basic subdivision paradigm have been proposed. These range from changing subdivision rules locally, as part of a global iterative process [Kobbelt 1996; Kobbelt and Schröder 1998; Diewald et al. 2002], to pulling and cutting the control net after sufficient refinement [Biermann et al. 2002]. A restriction of the designer's freedom was lifted without changing the basic simple subdivision paradigm in [Stam and Loop 2003]. This scheme combines [Catmull and Clark 1978] and [Loop 1987] with a $C^{1}$ transition rule, i.e. allowing the designer to freely place quadrilaterals and triangles (quads and tris) in the control net. [Levin and Levin 2003] even gives tools and an explicit scheme to join quad and tri subdivision $C^{2}$ across an (infinite) edge. 4-3 subdivision also combines quads and triangles but with different underlying subdivision rules. An example is shown below in Figure 2.
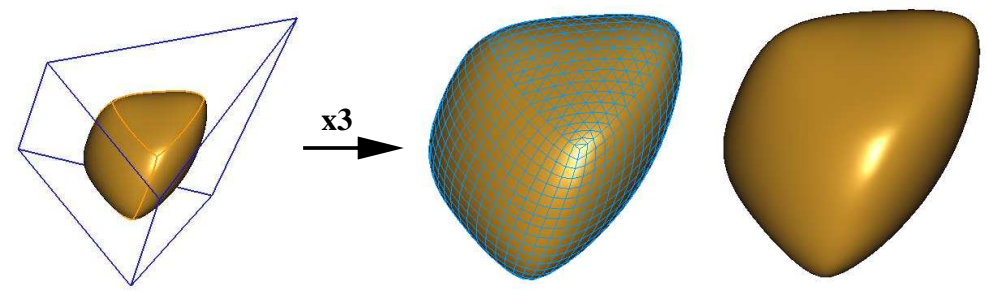

Fig. 2. 4-3 subdivision refines a mixed mesh of 4-sided and 3-sided facets. (left) Input control net and limit surface. (right two) Limit surface scaled 3 times with and without the refined control net.

A third, unwanted property is that, depending on the valence (= the number of neighbors) rather than the geometry, neighborhoods of points contract at different speeds. This lack of equi-contraction creates sharp or flat features in the refined tessellations as illustrated in Figure 3. The dual $C^{1}$ scheme [Doo and Sabin 1978] showed that equi-contraction, or, equivalently, sub-dominant eigenvalues of $\frac{1}{2}$ independent of valence, can be achieved without unduly complicating subdivision formulas.

4-3 subdivision matches the advantageous features both of [Stam and Loop 2003] and [Doo and Sabin 1978] by smoothing quad-tri meshes, up to second order in regular regions (in fact, with bounded curvature everywhere) and guaranteeing equi-contraction with simple rules. The scheme prevents undesirable ripples in diagonal directions without the drastic changes to the subdivision paradigm that separation of the mesh or iterative solution of variational problems represent: 4-3 subdivision is a combination of simple, fixed subdivision rules, that apply to a quad-tri mesh, avoid the diagonal artifacts of tensor-product 

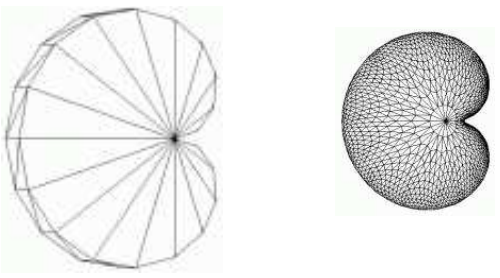

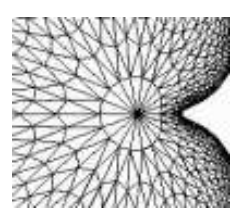

4-3
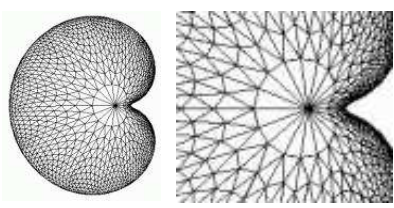

Loop

Fig. 3. Contraction of 4-3 subdivision by $1 / 2$ contrasted with Loop's subdivision.

subdivision and provide equi-contraction and bounded curvature everywhere, including 4-3 transitions from a quad to a tri net and at extraordinary nodes.

The 4-3 rule for transition between quad and triangular sub-meshes is simple, because both the quad and the tri bases of 4-3 subdivision are almost identical box-splines ${ }^{1}$ of degree four [de Boor et al. 1993]. Indeed, the matrices $\mathcal{A}_{3}$ and $\mathcal{A}_{4}$, whose rows indicate directions of averaging control points, differ in only one entry. By contrast, $\mathcal{A}_{3}$ and $\mathcal{A}_{\mathrm{CC}}$ differ markedly:
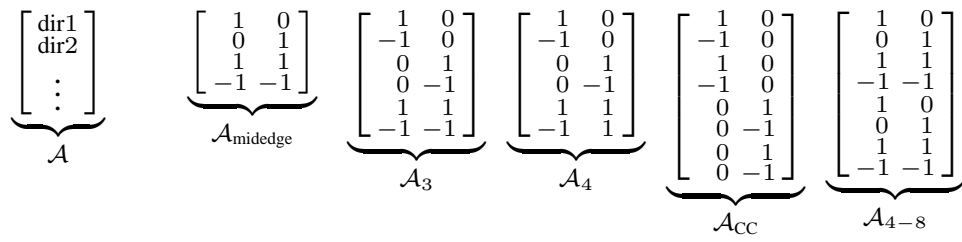

The box spline subdivision rules corresponding to $\mathcal{A}_{3}$ are the ones modified in [Loop 1987] to yield smoothing rules for general triangulations.

Choosing the box spline defined by $\mathcal{A}_{4}$, in place of $\mathcal{A}_{\mathrm{CC}}$ used in Catmull-Clark subdivision, requires some justification since the piecewise bicubic, tensor-product spline (NURBS) representation underlying Catmull-Clark subdivision is considered by some as the 'bedrock of geometric modeling'. First, in the scenario of interest, quadrilateral and triangular meshes are combined and therefore a uniform tensor-product representation is sacrificed ab initio. Second, the $\mathcal{A}_{4}$ spline is also a polynomial spline, in fact of lower degree than the bicubic $\mathcal{A}_{\mathrm{CC}}$ spline (see Figure 6 for its piecewise Bézier form) but just as smooth and has a smaller subdivision stencil! Finally, and this is a major point for graphics modeling, the $\mathcal{A}_{4}$ spline avoids undesirable ripples in more directions than tensor-product bicubic splines. The $\mathcal{A}_{4}$ directions yield $C^{2}$ box-splines on quadrilateral meshes. It may be viewed as a compromise between the not-so-smooth, $C^{1}$ box-spline corresponding to $\mathcal{A}_{\text {midedge }}$ of the simplest surface subdivision [Peters and Reif 1997; Habib and Warren 1999] and the overly smooth $C^{4}$ box spline $\mathcal{A}_{4-8}$ of 4-8 Subdivision [Velho and Zorin 2001] that doubles up the directions of $\mathcal{A}_{\text {midedge }}$. Each of these three representations, $\mathcal{A}_{\text {midedge }}, \mathcal{A}_{4}$ and $\mathcal{A}_{4-8}$ splines are linearly dependent or, from the modeling point of view, 'overcomplete'. That is, they can represent a given function with different sets of coefficients. Specifically,

${ }^{1}$ The fundamental box-spline subdivision algorithm in two variables inserts zeroes at the half-integer locations and scales the initial values at the integer locations by 4 , and then averages the values in the directions given by the $\mathcal{A}$ matrix. 
$\mathcal{A}_{4}$ box splines reproduce all degree three polynomials in two variables. But, in case the modeler does not manipulate the mesh directly but wants to fit the mesh by interpolation to given data, the modeler would have to select a(n energy) minimization criterion in addition to the position constraints to obtain a unique solution. Remarkably, by Lemma 3.2, $\mathcal{A}_{4}$ induces the unique symmetric $3 \times 3$ stencil (the size of the Catmull-Clark stencil) that preserves a constant ridge in any of the two Cartesian, axis-aligned and quincunx, diagonal directions.

This paper addresses two potentially distinct audiences. On one hand, the examples in Section 4 and the short statement of the algorithm in Section 2 are a guide for implementation and use of the scheme. On the other hand, Section 3 fully analyses the scheme with respect to shape and smoothness.

- The analysis of diagonal oscillations establishes $\mathcal{A}_{4}$ subdivision as the only one of its stencil-size to be free of ripples.

- The analysis of the 4-3 transitions largely follows [Levin and Levin 2003], but has a small modification that allows us to prove bounded curvature.

- The analysis at the eon proves $C^{1}$ continuity at an extraordinary point surrounded by an arbitrary combination of triangle- and quad-based subdivision schemes.

In the process, eigenvalues and eigenvectors are displayed that are helpful for the implementation of limit points and limit normals.

\subsection{Closely related literature}

The shape of Catmull-Clark subdivision surfaces was already analyzed in [Doo and Sabin 1978], globally faired in [Halstead et al. 1993], locally modified by explicit crease rules [DeRose et al. 1998], locally edited [Khodakovsky and Schröder 1999] and made to match prescribed normals by perturbation of the control net [Biermann et al. 2000]. The algorithm has been factored into micro-steps [Stam 2001; Zorin and Schröder 2001], modified to yield surfaces of bounded curvature [Sabin 1991] and blended with other rules to obtain continuous curvature [Prautzsch and Umlauf 1998; Biermann et al. 2000]. We will factor during the analysis (Section 3) and blend near extraordinary points (Section 4).

The midedge scheme's [Peters and Reif 1997; Habib and Warren 1999] 8-fold second largest eigenvalue $\frac{1}{4}$ for valence 3 motivates the exhaustive analysis of linear, stationary $C^{1}$ algorithms based on the Jordan canonical form in [Reif 1999]. Our analysis is largely based on the simpler analysis of standard cases in [Peters and Reif 1998] and [Umlauf 1999].

In [Iske et al. 2002], Malcolm Sabin started a list of shape artifacts of subdivision schemes and pointed out that the fractal support of the basis functions of various interpolatory subdivision schemes as well as $\sqrt{3}$ subdivision [Kobbelt 2000] implies that the sum of translates of the basis functions in any direction will exhibit ripples. 


\section{4-3 SUBDIVISION}

A non-boundary node of the control net is either

a regular node, i.e. a node surrounded by either four quadrilaterals or six triangles;

a 4-3 transition node, surrounded by two quadrilaterals followed by three triangles; or

an extraordinary node (short eon) converging to an extraordinary point (eop) under subdivision.

In each subdivision step, the number of eons stays constant, the number of boundary nodes and 4-3 transition nodes doubles and regular nodes quadruple. The algorithm is as follows.

Input: Control net with 3- and 4-sided facets.

Output: Control net with four times as many 3- and 4-sided facets.

The positions of new nodes are computed as a linear combination of the old nodes with weights summing to one. It is convenient to display the weights, without the normalization to one, as the stencils in Figure 4. For example, the rule (1a) in column (1) and row (a)

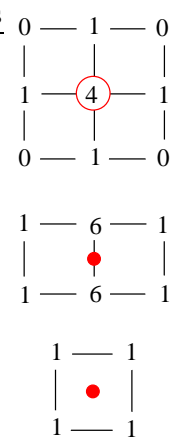

(1)
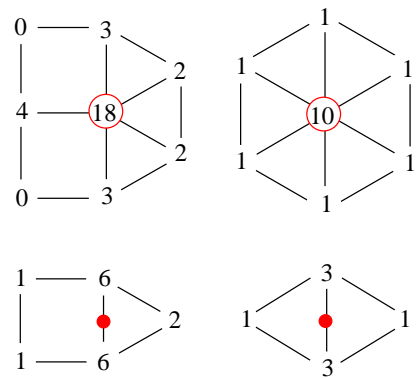

(2)

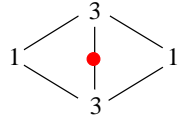

$(3)$

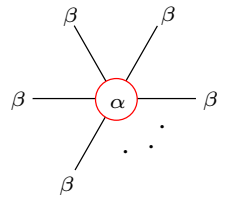

(a)

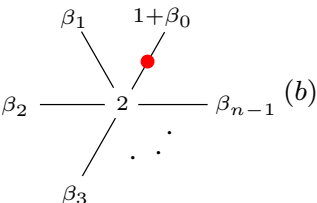

(4)

Fig. 4. Stencils for 4-3 subdivision: row (a) gives rules for new nodes replacing old nodes, row (b) show new nodes associated with edges and faces. The columns correspond to: (1) $\mathcal{A}_{4}$ subdivision, (2) 4-3 transition, (3) $\mathcal{A}_{3}$ subdivision, (4) eon subdivision (vertex with $n$ neighbors). Here $\beta_{i}:=\frac{1}{n}\left(1+2 \cos \frac{2 \pi i}{n}\right), \beta:=\frac{1-\alpha}{n}$ and $\alpha$ is free to choose in the interval $\left[\frac{1}{4} . \cdot \frac{3}{4}\right]$. The recommended values are $\alpha_{(n=3)}=3 / 8, \alpha_{(n=4)}=1 / 2$ and $\alpha_{(n>4)}=3 / 5$. Near eons, rule (4b) slowly takes over from rules (1b,2b,3b) as the subdivision level increases (see text).

says that the new position of a node surrounded by four quads is $\frac{4}{8}$ the old position plus $\frac{1}{8}$ of each direct neighbor. The circle indicates the logical correspondence of the new node to an old node. In row (b) a $\bullet$ indicates correspondence of the new node to an edge or facet. For example, (2b) second column, bottom determines the position of a new node on a 4-3 transition edge as $\frac{6}{16}$ times the neighbors on the edge, $\frac{2}{16}$ times the third triangle neighbor and $\frac{1}{16}$ times the remaining quad neighbors.

Note that new nodes on edges attached to an eon can be defined in two ways; by one of the regular rules (1b), (2b) or (3b), or, alternatively, by rule (4b). In the first subdivision steps $\sigma \leq \sigma_{0}$, say $\sigma_{0}=3$, we average $\frac{\sigma-1}{\sigma}$ times (4b) with $\frac{1}{\sigma}$ times the regular stencil. After subdivision $\sigma_{0}$, new nodes on edges attached to an eon only use (4b). All stencils are normalized to sum to 1 . For example, the weights of (4b) need to be scaled by $1 / 4$. 
Since (4b) moves nodes towards the affine image of a circle, this gradual introduction better preserves intended asymmetric features around the eon.

Boundary rules and crease rules are easily implemented (Figure 24) along the lines of [Biermann et al. 2000] and [DeRose et al. 1998].

\section{PROPERTIES}

For the analysis of 4-3 subdivision, it is convenient ${ }^{2}$ to break each subdivision step into two stages. First, the mesh is refined by adding nodes whose position is determined by (bi-)linear interpolation (see top row of Figure 5). Then the refined mesh is smoothed out by a second step that requires only one stencil for each mesh type. For example, lower left,
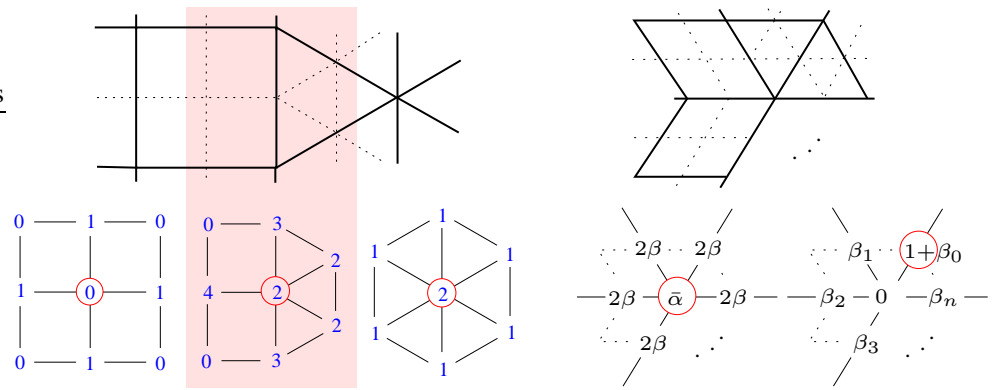

Fig. 5. Factored stencils for 4-3 subdivision. (Bi-)linear interpolation (refinement, top) followed by smoothing on the refined mesh (bottom). Here $\bar{\alpha}:=2 \alpha-1, \beta_{i}$ and $\beta$ as in Figure 4.

to compute the $\mathcal{A}_{4}$ box-spline node after bilinear interpolation, the node is replaced by the average of its four neighbors. By comparison, the smoothing stencil for Catmull-Clark subdivision after bilinear interpolation is $\left[\begin{array}{lll}1 & 2 & 1 \\ 2 & 4 & 2 \\ 1 & 2 & 1\end{array}\right] / 16$.

\subsection{Analysis at regular nodes}

According to [de Boor et al. 1993] (see also [Prautzsch and Boehm 2002; Warren and Weimer 2002]), a box-spline is defined by and can easily be analyzed via its matrix of convolution directions $\mathcal{A}$ : both $\mathcal{A}_{3}$ and $\mathcal{A}_{4}$ yield degree $4, C^{2}$ box-splines that reproduce all degree 3 polynomials in two variables. The sum of the entries in each column of the $\mathcal{A}$ matrix is even, and hence the shift of the box-spline mesh under subdivision is a multiple of the refined mesh spacing, a property often called 'primal'. The support or footprint of the $\mathcal{A}_{3}$ box-spline is hexagonal and the one of the $\mathcal{A}_{4}$ box-spline is octagonal. By comparison, the $\mathcal{A}_{\mathrm{CC}}$ spline is also primal, $C^{2}$ and reproduces all degree 3 polynomials, but is of degree 6 (bi-cubic) and therefore has the larger stencil and support shown below, on the right:

\footnotetext{
${ }^{2}$ For a run-time efficient implementation, such factoring is generally not a good idea, since the refined nodes now need to be visited twice and, without smart caching, two copies of the refined mesh have to be stored. This trade-off is a special case of a spectrum of trade-offs. One extreme, with a large stencil, is the pretabulation of the rules for limit points corresponding a fixed number of subdivision steps. The other extreme, with a minimal stencil, is the complete factorization into (box-spline) averaging steps. [Peters and Reif 1997; Stam 2001; Zorin and Schröder 2001].
}

ACM Transactions on Graphics, Vol. V, No. N, Month 20YY. 


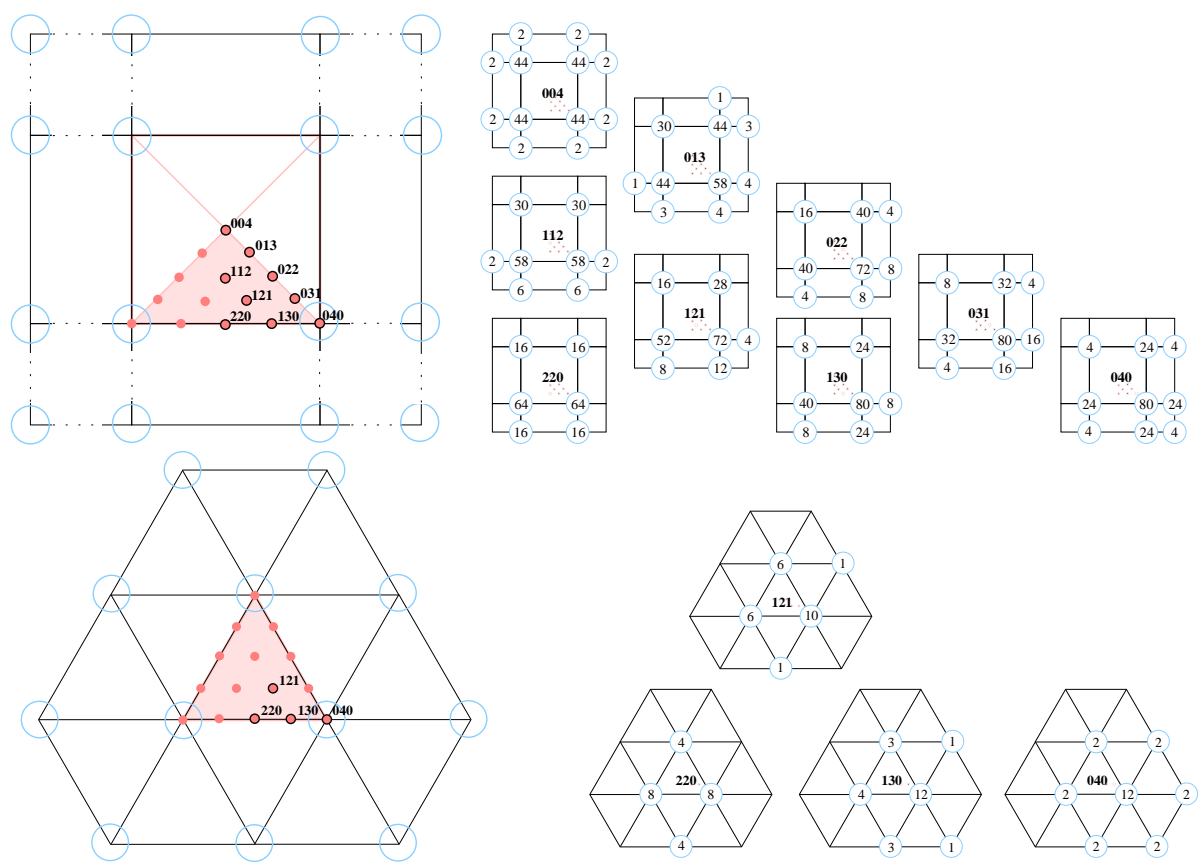

Fig. 6. Conversion from box-spline to Bézier form, for $\mathcal{A}_{4}$ (top) and $\mathcal{A}_{3}$ (bottom). The stencils (to be normalized to sum to 1) are shown on the right, the sub-meshes on the left show the logical position of the Bézier coefficients (represented by their subscripts on the shaded domain) with respect to the box-spline coefficients (represented as circles). The stencils on the right are arranged in the same logical position. Each lists the weights such that the weights times the corresponding box-spline coefficients yields the Bézier coefficient at that logical position. For example, the $\mathcal{A}_{4}$ Bézier coefficient with subscript 220 is $\frac{16}{192}$ times the four nonadjacent corner nodes plus $\frac{64}{192}$ times the two direct neighbors. The $\mathcal{A}_{3}$ Bézier coefficient with subscript 220 is $\frac{8}{24}$ times the nodes to the left and right, plus $\frac{4}{24}$ times the node above and below.

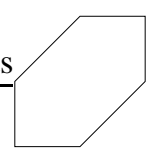

$\mathcal{A}_{3}$

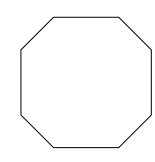

$\mathcal{A}_{4}$

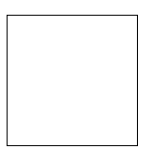

$\mathcal{A}_{C C}$

Figure 6 gives the change of basis for writing $\mathcal{A}_{3}$ and $\mathcal{A}_{4}$ splines in Bézier form. As mentioned before, $\mathcal{A}_{4}$ splines (as well as $\mathcal{A}_{\text {midedge }}$ and $\mathcal{A}_{4-8}$ ) have direction vectors that are not unimodular and hence the representation is overcomplete [de Boor et al. 1993]. Specifically, the two (bi-infinite) coefficient sequences

$$
\begin{aligned}
& -1+1-1+1 \\
& \begin{array}{llll}
0 & 0 & 0 & 0
\end{array} \\
& \begin{array}{llll}
+1 & -1 & +1 & -1
\end{array} \\
& \begin{array}{llll}
0 & 0 & 0 & 0
\end{array} \\
& -1+1-1+1 \\
& \begin{array}{llll}
0 & 0 & 0 & 0
\end{array} \\
& +1-1+1-1 \\
& \begin{array}{llll}
0 & 0 & 0 & 0
\end{array}
\end{aligned}
$$

both generate the zero function and oscillations that include the \pm 1 pattern on the left damp out. This creates both problems and advantages. Potential problems are that (P1) damping reduces a designers change to the surface; and (P2) fitting routines that rely on 

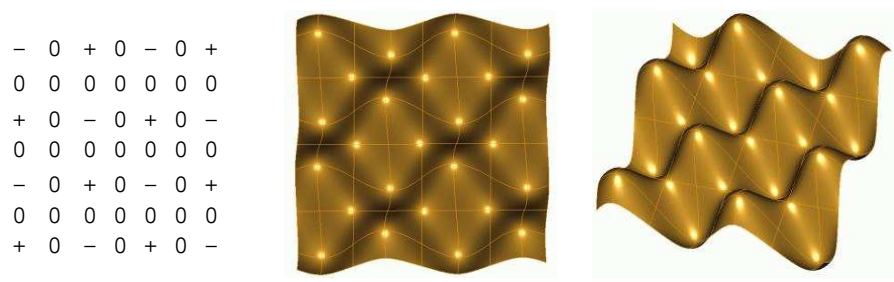

Fig. 7. A regular quad-grid with height values $\mathbf{+ 1 , 0} \mathbf{0}$ and $\mathbf{- 1}$ (left) results in the shape on the right. Conversely, the interpolant, minimizing the 2-norm of coefficients, to the data sampled from the surface on the right is the regular quad-grid with values $+1,0$ and -1 .

inverting point data do not have a unique solution. In the latter case, we need to select an approximant that best balances matching values and minimizing some 'energy'. Note that lack of uniqueness does not restrict the designer further. Since $\mathcal{A}_{4}$ reproduces polynomials of degree three, intentional oscillations can still be modeled. For example,the coefficient choice in Figure 7 will model the oscillations that might have been intended by the \pm 1 pattern above. Advantages of the overcompleteness are that (A1) designers have different options of expressing a given shape, and (A2) coefficients can be chosen to best match a certain application, say to reduce noise. For example, [Ron and Shen 1998] construct frames for wavelet decomposition from 4-direction box splines. The key advantage of using $\mathcal{A}_{4}$ is formalized in the following definition.

Definition 3.1. A subdivision scheme is ripple-free in the direction $\mathrm{d}$, if a control net with constant first differences in the direction d results in a surface whose first derivative in the direction $d$ is constant.

We prove that, among subdivision schemes with a small stencil size, the four-direction spline is unique in avoiding ripples. Note that, since not every $3 \times 3$ smoothing stencil corresponds to a box spline, differentiation of box splines can only establish the trivial direction of the proof: that 4-direction box spline subdivision preserves diagonal ridges. In fact, the proof eschews differentiation altogether and works only with differences.

LEMMA 3.2 (RIPPLE-FREE). $\mathcal{A}_{4}$-subdivision is the only subdivision with $3 \times 3$ stencil that is symmetric and ripple-free in all of the directions $\left[\begin{array}{l}1 \\ 0\end{array}\right],\left[\begin{array}{l}0 \\ 1\end{array}\right],\left[\begin{array}{l}1 \\ 1\end{array}\right],\left[\begin{array}{c}1 \\ -1\end{array}\right]$.

PROOF. The first and more difficult part of the proof is to prove uniqueness. It suffices to consider a finite section of an infinite, diagonally symmetric, shift invariant ridge pattern as shown on the left in the display below. Specifically, we normalize by subtracting a constant and scale so that the second off-diagonal layer is zero, the diagonal is 4 and $0 \leq \mathrm{a}<4$. Entries that do not enter the calculation are not shown. Since only one possible smoothing stencil preserves the ridges constant differences in the diagonal direction, we need not consider unsymmetric ridges at this point. Also, since all computations are local, it suffices to consider a finite section.

Due to symmetry, the $3 \times 3$ smoothing stencil has the form $\left[\begin{array}{ccc}\gamma & \beta & \gamma \\ \beta & \alpha & \beta \\ \gamma & \beta & \gamma\end{array}\right]$, where $\alpha+4 \beta+4 \gamma=$ ACM Transactions on Graphics, Vol. V, No. N, Month 20YY. 
1. and we compute

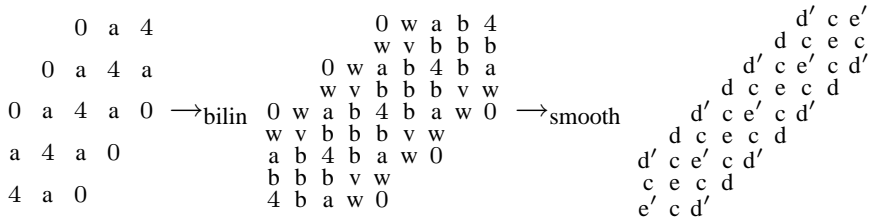

The values after bilinear refinement (middle) are $\mathrm{b}:=2+\frac{\mathrm{a}}{2}, \mathrm{v}:=1+\frac{\mathrm{a}}{2}, \mathrm{w}:=\frac{\mathrm{a}}{2}$. After smoothing, we have

$$
\begin{aligned}
\mathrm{e}-\mathrm{e}^{\prime} & =(\mathrm{b} \alpha+4 \beta \mathrm{b}+2(4+\mathrm{a}) \gamma)-(4 \alpha+4 \beta \mathrm{b}+2(\mathrm{~b}+\mathrm{v}) \gamma) \\
& =\alpha(4-\mathrm{b})+2 \gamma(\mathrm{b}-4+\mathrm{v}-\mathrm{a})=\alpha\left(2-\frac{\mathrm{a}}{2}\right)-2 \gamma .
\end{aligned}
$$

Since the diagonal remains monotone if and only if $\mathrm{e}=\mathrm{e}^{\prime}$ and the subdivision weights $\gamma$ and $\alpha$ do not depend on the data, here "a", we have $\alpha=0$ and $\gamma=0$ and hence $\beta=\frac{1}{4}$, i.e. the $\mathcal{A}_{4}$ rule.

Conversely, any unsymmetric ridge with profile (traversal cut) height $\left\langle y_{-2}, y_{-1}, y_{0}, y_{1}, y_{2}, y_{3}\right\rangle$ can be decomposed into the linear function $(1-t) y_{-2}+t y_{3}$ and the two symmetric ridges

$$
\begin{array}{rlrl}
\left\langle 0, z_{1}, z_{2}, z_{1}, 0,0\right\rangle, & z_{1}:=-\frac{4}{5} y_{-2}+y_{-1}-\frac{1}{5} y_{3}, & z_{2}:=-\frac{2}{5} y_{-2}+y_{0}-y_{2}+\frac{2}{5} y_{3} ; \\
\left\langle 0,0, z_{3}, z_{4}, z_{3}, 0\right\rangle, \quad z_{3}:=-\frac{1}{5} y_{-2}+y_{2}-\frac{4}{5} y_{3}, \quad z_{4}:=\frac{2}{5} y_{-2}-y_{-1}+y_{1}-\frac{2}{5} y_{3} .
\end{array}
$$

It is therefore sufficient to consider symmetric ridges. If $\beta=\frac{1}{4}$ and $\alpha=\gamma=0$, we have $\mathcal{A}_{4}$ subdivision and $\mathrm{c}:=\frac{7}{4}+\frac{\mathrm{a}}{2}$, and $\mathrm{d}=\mathrm{d}^{\prime}:=1+\frac{\mathrm{a}}{2}$. We subtract $\mathrm{d}$ and scale to 4 to restore the initial ridge pattern and establish invariance. Invariance of axis-aligned ridge in the other three directions is similarly verified and the smoothness of the box-spline then translates constant differences into constant first derivatives.

The box splines corresponding to $\mathcal{A}_{\text {midedge }}$ and $\mathcal{A}_{4-8}$ are also based on four directions of averaging. $\mathcal{A}_{\text {midedge }}$ has the best shape preservation due to its minimal stencil, but is only $C^{1}$. By contrast, $\mathcal{A}_{4-8}$ is $C^{4}$ apart from eons and that smears out features. Ripplefreeness of $\mathcal{A}_{3}$ along its three averaging directions follows readily from the symmetries of its stencil. The stencil entries are not unique since any radially symmetric stencil will do. Lemma 3.2 shows that we cannot hope for ripple-freeness of $\mathcal{A}_{3}$ in the direction $[1,-1]$.

\subsection{4-3 transition}

The key to properties of the subdivision surface at a point is the local subdivision matrix $\mathrm{S}$ that maps the control net $\mathrm{X}^{m}$ of the $m$ th level of subdivision to $\mathrm{X}^{m+1}$ at level $m+$ 1: $\mathrm{X}^{m+1}=\mathrm{SX}^{m}$ [Doo and Sabin 1978; Reif 1999] The analysis of the transition from a quad to a tri net is both more complex and simpler than the analysis at an eon: on one hand, the neighborhood of a line segment needs to be analyzed, as opposed to just a single point; on the other hand, the subdivision matrix at a transition node, $\mathrm{S}_{43}$, is easily determined, on a unit-spaced subnet $\mathrm{X}$ corresponding to $[-3,3] \times[-3,3]$. The eigenvalues of this subdivision matrix, ordered by modulus, are $1, \frac{1}{2}, \frac{1}{2}, \frac{1}{4}, \frac{1}{4}, \frac{1}{4}, \ldots$. The stencil of the left eigenvector corresponding to the eigenvalue 1 is shown in Figure 8, left. Normalized so that they sum to one, the stencil weights multiply the corresponding mesh points to yield the limit point on the 4-3 transition. The two left eigenvectors of the eigenvalue $\frac{1}{2}$ 
yield the tangent direction stencils shown in Figure 8, right. Computing the two linear combinations (summing to zero) yields two vectors whose cross product is the normal at the limit point. The two right eigenvectors to the eigenvalue $\frac{1}{2}$ are plotted as an $(x, y)$ -
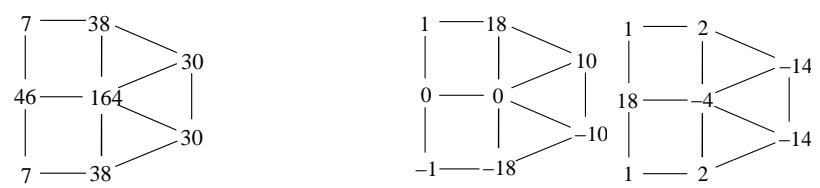

Fig. 8. Left eigenvector stencils corresponding to eigenvalues $1, \frac{1}{2}, \frac{1}{2}$.

pair in Figure 9. The $y$-axis is the 4-3 transition line. Analogous to the characteristic map [Reif 1995], the eigenvectors span a characteristic strip of functions on either side of the transition line. The domain of these two functions is indicated as shaded quads and

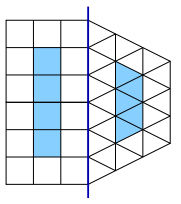

Fig. 9. Right eigenvectors corresponding to eigenvalues $\frac{1}{2}, \frac{1}{2}$ displayed as $(x, y)$-pair.

triangles. By transforming them into Bézier form with the help of the formulas in Figure 6, the functions are easily shown to be regular and injective. The eigenvectors corresponding to the eigenvalue $\frac{1}{4}$ correspond to elliptic and hyperbolic eigenfunctions as required of a fexible scheme [Peters and Reif 2004].

To analyze the continuity across a whole segment of the 4-3 transition, we apply the ideas of [Levin 2003; Rioul 1992] and largely follow the explicit procedure given in Section 3 of [Levin and Levin 2003]:

(1) Select a (minimal) subset $L$ of nodes that define, via subdivision, a segment of the transition curve between two nodes.

(2) On $L$, determine the subdivision matrices $\mathrm{S}_{43}$ and $\mathrm{S}^{+}$for either endpoint of the segment.

$(3,4)$ Apply to $\mathrm{S}^{+}$a similarity transformation that diagonalizes $\mathrm{S}_{43}$ (e.g. using the eigenvectors $V$ of $\mathrm{S}_{43}$ ).

(5) To prove $C^{m}$ continuity, remove the first $\left(\begin{array}{c}m+2 \\ 2\end{array}\right)$ rows and columns of the transformed matrices to get submatrices $Y$ and $Y^{+}$. Bound the joint spectral radius of $Y$ and $Y^{+}$above by the decreasing sequence in $k$ :

$\rho^{[k]}\left(Y, Y^{+}\right):=\left(\max \left\{\left\|Z_{k} Z_{k-1} \cdots Z_{1}\right\|_{\infty}: Z_{i} \in\left\{Y, Y^{+}\right\}, i=1 . . k\right\}\right)^{1 / k}$.

In [Levin and Levin 2003], the procedure verifies rules for a $C^{2}$ transition between $\mathcal{A}_{3}$ and $\mathcal{A}_{\mathrm{CC}}$ splines based on second-order quasi-interpolants. Since the second-order quasiinterpolant for $\mathcal{A}_{4}$ and $\mathcal{A}_{\mathrm{CC}}$ splines are identical, namely $Q f:=f-\left(D^{02}+D^{20}\right) f / 6$, we could adapt those rules to obtain a $C^{2}$ transition between $\mathcal{A}_{4}$ and $\mathcal{A}_{3}$ splines. We chose not to because we do not want to have special rules for nodes adjacent to 4-3 transition 
edges. Modifying adjacent layers makes the code slightly more complex and the analysis near the eons considerably more complex. (The analysis of eons is not a concern in [Levin and Levin 2003]). Since the second-order quasi-interpolant for $\mathcal{A}_{4}$ and $\mathcal{A}_{\mathrm{CC}}$ splines are identical, the argument in [Stam and Loop 2003] shows that $y^{2}$ is not reproduced by the 4-3 transition rules. Figure 10 illustrates this in terms of sectional curvature. Nevertheless, we can prove first-order smoothness and boundedness of the curvature.

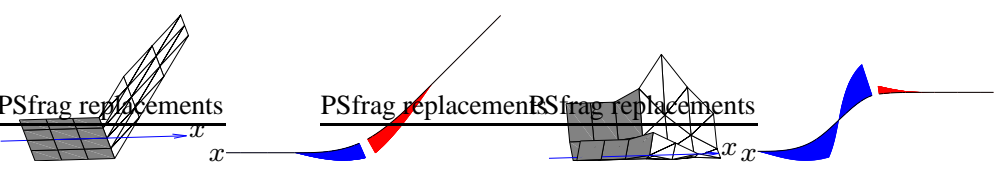

Fig. 10. Control nets and corresponding sectional curvature in the $x$ direction.

LEMMA 3.3 (SMOOTHNESS ACROSS 4-3 TRANSITIONS). Across 4-3 transitions, excluding degenerate control nets and eons, 4-3 subdivision surfaces are $C^{1}$ and have bounded curvature.

PROOF. We recall that the eigenvalues of $S_{43}$ are $1, \frac{1}{2}, \frac{1}{2}, \frac{1}{4}, \frac{1}{4}, \frac{1}{4}, \frac{1}{8}, \ldots$ and that constant and linear functions are reproduced by the eigenfunctions corresponding to $1, \frac{1}{2}, \frac{1}{2}$. This implies $C^{1}$ continuity and bounded curvature at every limit point corresponding to an original mesh node. To make statements about the points in between, along the 4-3 transition curve, we need to analyze the pair of matrices $\mathrm{S}_{43}$ and $\mathrm{S}^{+}$. $\mathrm{S}^{+}$is determined just like $\mathrm{S}_{43}$ but with the indices of the nodes modified by the subdivision matrix shifted by one in the $y$ direction. All combinations of these two matrices generate the points on a segment $x=0, y=[0 . .1]$ of 4-3 transition.

We essentially apply to $\mathrm{S}^{+}$the eigen-decomposition $V^{-1} \mathrm{~S}_{43} V$ that diagonalizes $\mathrm{S}_{43}$. Since $V \in \mathbb{R}^{56 \times 56}$ corresponding to the integer grid $L:=[-3,3] \times[-3,4]$ is not of full rank, we form $V$ from the six eigenvectors corresponding to the six leading eigenvalues plus the nullspace of the eigenvectors. This yields

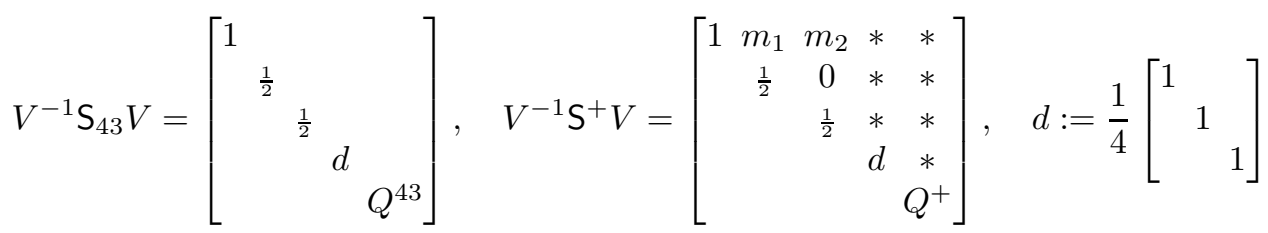

where $Q^{43}$ and $Q^{+}$are of size $50 \times 50$. To establish $C^{1}$ continuity, step 5 of the recipe of [Levin and Levin 2003] suggests computing

$$
\rho^{[k]}\left(Y^{43}, Y^{+}\right):=\left(\max \left\{\left\|Y^{\epsilon_{k}} Y^{\epsilon_{k-1}} \cdots Y^{\epsilon_{1}}\right\|_{\infty}: \epsilon_{i} \in\{43,+\}, i=1 . . k\right\}\right)^{1 / k},
$$

for increasing $k$ and

$$
Y^{43}:=\left[\begin{array}{cc}
d & 0 \\
0 & Q^{43}
\end{array}\right], \quad Y^{+}:=\left[\begin{array}{ll}
d & * \\
0 & Q^{+}
\end{array}\right] .
$$

This yields a decreasing sequence and the spectral radius $\rho\left(Y^{43}, Y^{+}\right)$is equal to $\lim _{k} \sup \rho^{[k]}\left(Y^{43}, Y^{+}\right)$. However, convergence is poor and, due to numerical roundoff, 
this approach would at best yield an upper bound, close to but above the number 0.25 that is required to show boundedness of the second derivative. So, instead, we observe that

$$
Y^{\epsilon_{k}} Y^{\epsilon_{k-1}} \cdots Y^{\epsilon_{1}}=\left[\begin{array}{cc}
d^{k} & * \\
0 & \tilde{Q}
\end{array}\right] \quad \tilde{Q}:=Q^{\epsilon_{k}} Q^{\epsilon_{k-1}} \cdots Q^{\epsilon_{1}}, \epsilon_{i} \in\{43,+\}, i=1 . . k .
$$

Since we know $d$ exactly, we can simply compute a decreasing upper bound on the joint spectral radius of $Q^{43}$ and $Q^{+}$. Already ${ }^{3} \rho^{[11]}\left(Q^{43}, Q^{+}\right)$is less than 0.25 and therefore $\rho\left(Y^{43}, Y^{+}\right)=\max (d)=0.25$. For the individual component functions $\rho\left(Y^{43}, Y^{+}\right)<$ 0.5 implies $C^{1}$ continuity and since the Hölder continuity of the derivatives is $-1-$ $\log _{2}\left(\rho\left(Y^{43}, Y^{+}\right)\right)=1$, the second derivative and hence the curvature are bounded. Since we are dealing with parametric manifolds, we have to additionally concern ourselves with the injectivity of the characteristic strip, (which is not a consideration in the Levin procedure). Above, injectivity of the characteristic strip has only been claimed for the limit points of original transition nodes. However, looking at the structure of $V^{-1} \mathrm{~S}^{+} V$ in detail, the eigenvectors of $\mathrm{S}^{+}$corresponding to $\frac{1}{2}$ are simply $v_{1}+m_{1} v_{0}$ and $v_{2}+m_{2} v_{0}$ where $v_{i}$ is the eigenvector of $\mathrm{S}_{43}$ corresponding to the $i$ th eigenvalue, ordered by size. That is the characteristic strip is simply shifted and hence the injectivity is inherited.

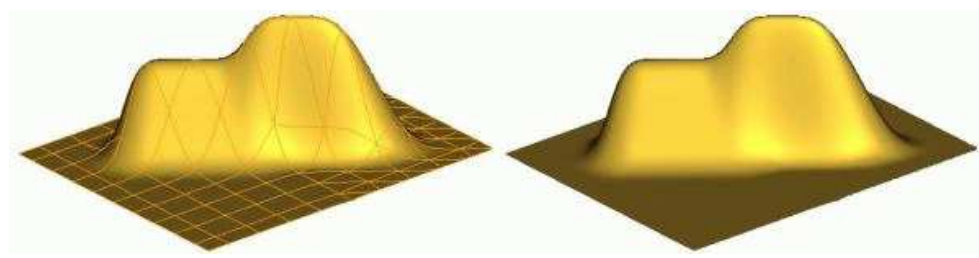

Fig. 11. A straight ridge across the quad-tri transition edge (the quad-diagonal is continued into the triangle mesh) with control points at equal height is marked by a difference in height.

Since the $\mathcal{A}_{3}$ and the $\mathcal{A}_{4}$ box spline are of different height, the height of a ridge changes if we choose the same control point height on both sides and for the 4-3 transition node (Figure 11). So why do we not observe height variations across the 4-3 interface, say in Figure 19? The answer is that both $\mathcal{A}_{3}$ and $\mathcal{A}_{4}$ splines and their average, the 4-3 transition rule, reproduce linear functions. Setting, say, three parallel ridges to 1 results in the same height along the ridge across the 4-3 transition.

\subsection{Extraordinary points and nodes}

Due to the arbitrary combination of triangles and quadrilaterals among the $n$ facets that surround an eon, the analysis of 4-3 subdivision faces two fundamental technical difficulties not encountered in the standard, symmetric setting. First, we cannot (directly) apply discrete Fourier transform techniques. Second, the $n$ surface pieces, that join to form an annulus surrounding the eon (Figure 13, left), are not piecewise polynomial unless the facets are either all triangles or all quads. Remarkably, a full analysis of the spectrum and the leading eigenvectors is nevertheless possible.

\footnotetext{
${ }^{3}$ To get faster convergence, it pays to use the first 10 eigenvectors, carefully omitting the dependent one. 

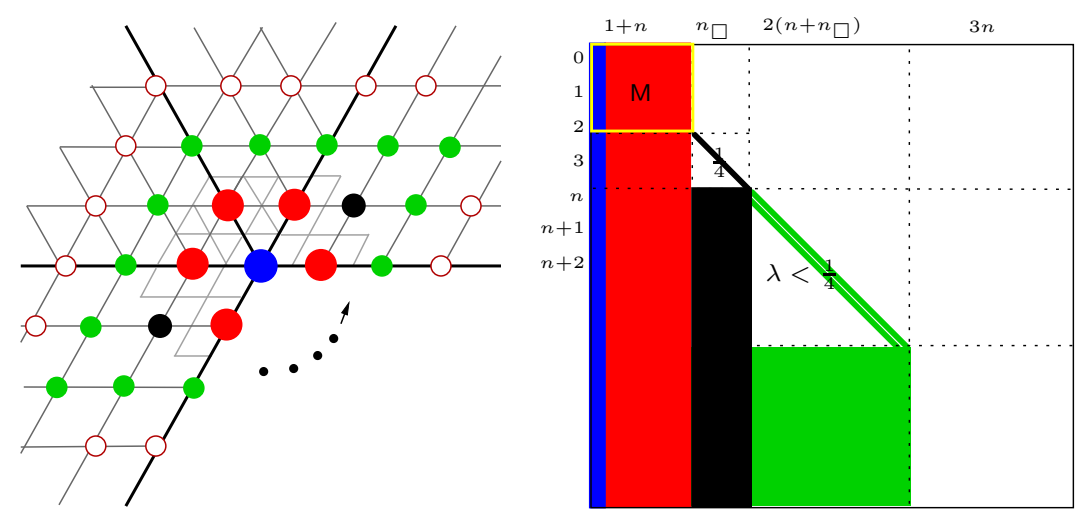

Fig. 12. Correspondance of the submesh surrounding an eon (extraordinary node, left) and the column structure of the subdivision matrix S (right). White areas in S correspond zero entries. The nodes represented as hollow circles in the third ring (left) are needed to define a consistent surface annulus (see Figure 13); but they do not contribute to the submesh that defines the refined annulus.

The $s \times s$ local subdivision matrix $\mathrm{S}$ transforms a portion of the net, surrounding the eon, to an isomorphic net. To be useful for analysis, this local net must be large enough to define a consistent surface annulus: that is the union of the annulus and its images scaled by $\left(\frac{1}{2}\right)^{k}, k=1, \ldots, \infty$ covers the neighborhood of the eop and consecutive annuli join without overlap. Let $n$ be the total number of facets surrounding the eon and $n_{\square} \leq n$ the number of the quads among these facets. Then $s:=1+6 n+3 n_{\square}$ is sufficient to define a consistent surface annulus for analyzing the eigenstructure. (The corresponding mesh nodes are shown as bullets in Figure 13, left; since the direct neighbors of the eon have special rules, one more ring has to be selected to write out annuli of the surface in Bézier form.) The nodes can be ordered to yield a largely lower-diagonal S shown in Figure 12, right: Apart from the submatrix $\mathrm{M}$, the diagonal entries are eigenvalues, and, in decreasing order, $\frac{1}{4}, \frac{1}{8}, \ldots, 0$. The number of $\frac{1}{4}$ eigenvalues equals $n_{\square}$ and hence may be zero. The properties of $\mathrm{S}$ depend crucially on those of $\mathrm{M}$. M is defined in terms of the $n \times n$ matrices $\mathrm{E}, \mathrm{C}$ and I with entries

$$
\mathrm{E}_{i j}:=\frac{1}{n}, \mathrm{C}_{i j}:=\frac{2}{n} \cos \frac{2 \pi(i-j)}{n}, \mathrm{I}_{i j}:= \begin{cases}1 & \text { if } i=j \\ 0 & \text { else, }\end{cases}
$$

and the $n \times 1$ vector e defined by $\mathrm{e}_{i}:=1$. The following is easy to check.

LeMma 3.4. $\mathrm{E}+\mathrm{C}$ is a projection, i.e. $(\mathrm{E}+\mathrm{C})^{2}=\mathrm{E}+\mathrm{C}$ and

$$
\mathrm{C}^{2}=\mathrm{C}, \mathrm{Ce}=0, \mathrm{ee}^{t}=n \mathrm{E}, \mathrm{e}^{t} \mathrm{e}=n, \mathrm{Ee}=\mathrm{e}, \mathrm{EE}=\mathrm{E} .
$$

We also note that $\mathrm{C}$ is of rank 2 and $\mathrm{E}+\mathrm{C}$ is of rank 3 , i.e. $\mathrm{E}+\mathrm{C}$ projects $n$ points into a plane. The simple eigenstructure of

$$
\mathrm{M}:=\left[\begin{array}{cc}
(1-\alpha) & \frac{\alpha}{n} \mathrm{e}^{t} \\
\mathrm{e} / 2 & \frac{1}{4}(\mathrm{I}+\mathrm{E}+\mathrm{C})
\end{array}\right]
$$

is the key to the analysis. 
LemMa 3.5 (EIgENVALUeS OF M). For $0<\alpha<1$, M has the characteristic polynomial $\operatorname{det}(\mathrm{M}-\lambda \mathrm{Id})=(\lambda-1)\left(\lambda-\frac{1}{2}\right)^{2}\left(\lambda-\frac{1}{4}\right)^{n-3}\left(\lambda-\left(\frac{1}{2}-\alpha\right)\right)$.

PROOF. For $n=3$ the characteristic polynomial is easily computed and matches the claim. For $n \geq 4$, the rules of Lemma 3.4 yield $\mu(\mathrm{M})=0$, where

$$
\mu(x):=(x-1)\left(x-\frac{1}{2}\right)\left(x-\frac{1}{4}\right)\left(x-\left(\frac{1}{2}-\alpha\right)\right) .
$$

That is, $\mu$ is an annihilating polynomial for $\mathrm{M}$, and therefore $\lambda \in\left\{1, \frac{1}{2}, \frac{1}{4}, \frac{1}{2}-\alpha\right\}$ for any eigenvalue $\lambda$ of M. The multiplicity of the eigenvalues then equals the rank deficiency of $\mathrm{M}-\lambda \mathrm{Id}$.

If $\alpha \neq \frac{1}{4}$, we show that the eigenvalues $1, \frac{1}{2}$ and $\frac{1}{2}-\alpha$ have multiplicity 1,2 and 1 , respectively. The eigenvalue $\frac{1}{4}$ must then account for the remaining $n-3$ roots. By elementary row and column operations,

$$
\begin{aligned}
\operatorname{rank} & {\left[\begin{array}{cc}
(1-\alpha)-\lambda & \frac{\alpha}{n} \mathrm{e}^{t} \\
\frac{1}{2} \mathrm{e} & \left(\frac{1}{4}-\lambda\right) \mathrm{I}+\frac{1}{4}(\mathrm{E}+\mathrm{C})
\end{array}\right] } \\
& =\delta_{\lambda \neq 1 / 2}+\operatorname{rank}[(1-4 \lambda) \mathrm{I}+\mathrm{C}]
\end{aligned}
$$

where $\delta_{\lambda \neq 1 / 2}$ is 1 if $\lambda \neq 1 / 2$ and 0 else. Since $(1-4 \lambda) I+C$ is a circulant matrix, its $j$ th eigenvalue is $\begin{cases}2-4 \lambda & \text { if } j=1, \\ 3-4 \lambda & \text { if } j=-1, \\ 1-4 \lambda & \text { else. }\end{cases}$

For $\lambda=1$ and $\lambda=\frac{1}{2}-\alpha$, the matrix $(1-4 \lambda) \mathrm{I}+\mathrm{C}$ is of full rank $n$ since all eigenvalues are nonzero, while, for $\lambda=\frac{1}{2}$, the rank is $n-1$ as claimed.

If $\alpha=\frac{1}{4}$ then

$$
\operatorname{rank}\left(\mathrm{M}-\frac{1}{4} \mathrm{Id}\right)=\operatorname{rank}\left[\begin{array}{cc}
\frac{1}{2} & \frac{1}{4 n} \mathrm{e}^{t} \\
\frac{1}{2} \mathrm{e} & \frac{1}{4}(\mathrm{E}+\mathrm{C})
\end{array}\right]=1+\operatorname{rank} \mathrm{C}=3,
$$

and the multiplicity of the eigenvalue $\lambda=\frac{1}{4}$ is $n-2$.

Remark: If we weigh the projection $\mathrm{E}+\mathrm{C}$ with $1-\epsilon$ and the interpolation I with $1+\epsilon$ for $\epsilon \in[-1 . .1]$ then the eigenvalues of $M$ are in the set $\left\{1, \frac{1}{2}, \frac{1-\epsilon}{4}, \frac{1}{2}-\alpha\right\}$; that is, for $\epsilon \neq 0$, in the absence of other $1 / 4$ terms, the curvature would be either diverging or zero.

LEMMA 3.6 (EIGENVECTORS OF M). The leading left and right eigenvectors $1^{0}, r^{0}$ corresponding to the eigenvalue 1 and $1^{j}, \mathbf{r}^{j}, j=1,2$, corresponding to the eigenvalue $\frac{1}{2}$ are

$$
\mathrm{r}^{0}:=\left[\begin{array}{l}
1 \\
\mathrm{e}
\end{array}\right], \mathrm{r}^{1}:=\left[\begin{array}{l}
0 \\
\mathrm{c}
\end{array}\right], \mathrm{r}^{2}:=\left[\begin{array}{l}
0 \\
\mathrm{~s}
\end{array}\right], \mathrm{I}^{0}:=\left[n, 2 \alpha \mathrm{e}^{t}\right], \mathrm{I}^{j}=\left(\mathrm{r}^{j}\right)^{t},
$$

where the ith entry of $\mathrm{c}$ is $\mathrm{c}_{i}:=\cos \left(\frac{2 \pi}{n} i\right)$ and the ith entry of $\mathrm{s}$ is $\mathrm{s}_{i}:=\sin \left(\frac{2 \pi}{n} i\right)$.

Proof. With Id the $n+1 \times n+1$ identity matrix, it is easy to check that $\mathrm{I}^{0}(\mathrm{M}-\mathrm{Id})=$ $0=(M-I d) r^{0}$. Since $(E+C-I) c=0$ and $(E+C-I) s=0$ and $(E+C-I)^{t}=E+C-I$, also $\mathrm{I}^{j}(\mathrm{M}-\mathrm{Id} / 2)=0=(\mathrm{M}-\mathrm{Id} / 2) \mathrm{r}^{j}$ for $j=1,2$.

Since the entries of $\mathrm{S}$ to the right of $\mathrm{M}$ are zero, the left eigenvectors of $\mathrm{S}$ are simply those of $\mathrm{M}$ followed by $s-n-1$ zeros. Let $\mathrm{V}_{i}$ be the $i$ th neighbor node of the eon and $\mathrm{v}:=\left[0, \mathrm{v}_{0}, \ldots \mathrm{v}_{n-1}, 0, \ldots, 0\right]$. The practical implication is that we can compute the ACM Transactions on Graphics, Vol. V, No. N, Month 20YY. 


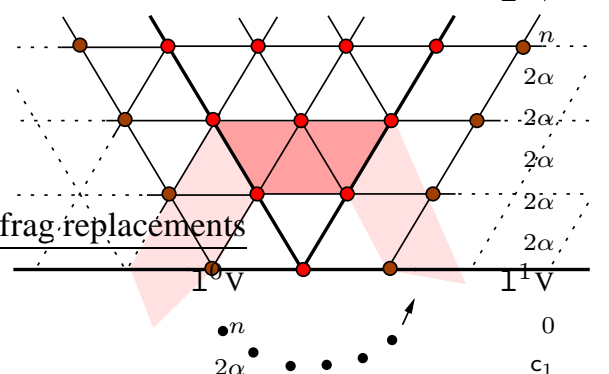

Fig. 13. Extended sector: control net (see row 3 column 2 of Figure 15) and, shaded darker, a sector of the characteristic map.

position of the eop as $1^{0} \mathrm{~V}$ and the normal direction at the eop as $1^{1} \mathrm{~V} \times \mathrm{I}^{2} \mathrm{~V}$ by applying the stencils:
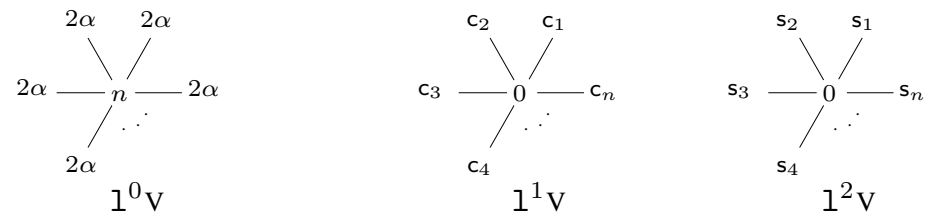

We now prove the $C^{1}$ continuity of the surface at the eop. Since the box-splines and the 4-3 transition are $C^{1}$ and the eigenvalues of $S$ decrease in the correct progression, we only need to show that the characteristic map

$$
\phi:\{1,2, \ldots, n\} \times \omega \subset \mathbb{R}^{n \times 2} \rightarrow \mathbb{R}^{2}, \quad \omega:=\text { convex hull }\left(\left[\begin{array}{l}
1 \\
0
\end{array}\right],\left[\begin{array}{l}
2 \\
0
\end{array}\right],\left[\begin{array}{l}
0 \\
2
\end{array}\right],\left[\begin{array}{l}
0 \\
1
\end{array}\right]\right) .
$$

is regular and injective onto a consistent planar annulus [Peters and Reif 1998] ${ }^{4}$. The characteristic map $\phi$ is defined by applying subdivision to the 2D control net $\left(r^{1}, r^{2}\right)$ where, for $k=1,2, r^{k}$ is a subdominant right eigenvectors of $\mathrm{S}$ corresponding to the eigenvalue $\frac{1}{2}$. Since, apart from $M$, the matrix $S$ is lower-diagonal (see Figure 12), and since Lemma 3.3 yields the first $n+1$ entries $\mathrm{r}^{k}(\ell)=\mathrm{r}^{k}(\ell)$ for $\ell=1, \ldots, n+1$, we can backsolve for the remaining entries of $\mathrm{r}^{k}$ in terms of the symbols $\mathrm{c}_{i}$ and $\mathrm{s}_{i}$. Then we look at three consecutive sectors, each corresponding to $\mathcal{A}_{3}$ or $\mathcal{A}_{4}$ subdivision. There are eight possible combinations and only two are redundant due to symmetry.

For each triple, we back-solve, using a symbolic solver such as Maple, and obtain the nodes corresponding to one extended sector of nodes. An extended sector of nodes consists of the nodes of the sector plus one layer of nodes from either neighbor sector (Figure 13). Without loss of generality, after rotation and uniform scaling in both directions, we may in the following assume that

(A) The node with index $(1,0)$ is $d_{0}:=\left[\begin{array}{l}1 \\ 0\end{array}\right]$ and $(0,1)$ corresponds to $d_{1}:=\left[\begin{array}{l}c_{1} \\ s_{1}\end{array}\right]$.

By substituting $\cos \left(\frac{2 \pi}{n} i\right)$ for $\mathrm{c}_{i}$ and $\sin \left(\frac{2 \pi}{n} i\right)$ for $\mathrm{s}_{i}$ for various $n$, we can draw the extended sectors in Figure 15. Thanks to the small stencils of the scheme, such an extended sector

${ }^{4}$ Note that the trapezoidal domain $\omega$ is the same for $\mathcal{A}_{3}$ and $\mathcal{A}_{4}$ subdivision. As can be seen from Figure 6 , for an eon entirely surrounded by triangles $\omega$ maps to three polynomial pieces; for an eon entirely surrounded by quads it maps to six polynomial pieces. 

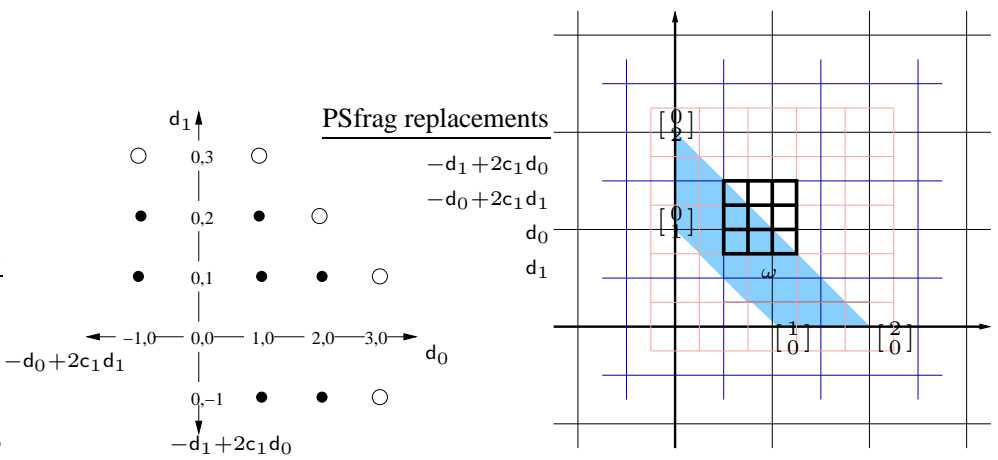

Fig. 14. (left:) Node indexing. Nodes represented by $\circ$ are needed for defining the characteristic map $\phi_{i}$ only if the corresponding sector is a quad. (right:) Domain grid after two refinements. One $4 \times 4$ subnet of 21 is emphasized at $(7 / 8,9 / 8)$.

completely defines the restriction $\phi_{k}$ of $\phi$ to the $k$ th domain $\omega$ :

$$
\phi_{k}: k \times \omega \rightarrow \Omega_{k} \subset \mathbb{R}^{2}, \quad(k, u, v) \mapsto(x, y)=\phi(k, u, v) .
$$

We can now look at the (between 16 and 21) coefficients that define each type of $\phi_{k}$. For all 8 configurations and all $n, j \in\{-1,0,1\}$ and $i \in\{0,1,2,3\}$, we verify by inspection (or range arithmetic) for $c_{1} \in\left[-\frac{1}{2} . .1\right]$ and $s_{1} \in\left[-\frac{1}{2} . .1\right]$ the following (cf. Figure 14).

(S1) The $y$-component of nodes with index $(i, j)$ is $j$.

(S2) The $x$-component of nodes with index $(i, j)$ is monotonically increasing.

After two subdivision steps (in symbols $c_{i}$ and $s_{i}$ ), we arrive at the refined mesh shown

in Figure 14, right. For every $4 \times 4$ subnet whose central quad overlaps the domain, we compute the differences in the two axis directions, i.e. the $i$-differences $b_{i+1, j}-b_{i, j}$ and the $j$-differences $b_{i, j+1}-b_{i, j}$.

(S3) All $i$-differences have a positive $x$ component and all $j$-differences have a positive $y$ component. (This is not true without subdivision!)

We scale the $i$-differences so that they have the form $\left[\begin{array}{c}1 \\ \delta_{1}\end{array}\right]$ and the $j$-differences as $\left[\begin{array}{c}\delta_{0} \\ 1\end{array}\right]$ (sketched as circles and boxes in Figure 16). For the range of $c_{1}$ and $s_{1}$, we check that the following holds.

(S4) For all subnets not overlapping the boundary of the sector, $\left|\delta_{i}(n)\right|<1$, i.e. no convex combination of $j$-differences is collinear with a convex combination of $i$-differences. For subnets overlapping the sector boundary, we have to compute some differences as averages of differences to account for 4-3 transitions and 3-4 transitions. Then, just as for interior subnets, the differences fall into two half-cones that only overlap at the origin as illustrated in Figure 16).

Finally, converting to Bézier form in the interior away from the sector boundaries, and looking at the recursion as one sector boundary is approached, we find (S5) along the boundary $E:=\left[\begin{array}{l}0 \\ 1\end{array}\right] . .\left[\begin{array}{l}1 \\ 0\end{array}\right]$ of $\omega$, subdivision generates polynomial curve segments that are monotone in the direction $\left[\begin{array}{c}1 \\ -1\end{array}\right]$ (i.e. do not fold back). With these observations, we can prove the main result.

THEOREM 3.7 (GLOBAL SMOOTHNESS AND CURVATURE BOUNDEDNESS). If $\frac{1}{4} \leq \alpha \leq$ $\frac{3}{4}$ then, excluding degenerate control nets, 4-3 subdivision surfaces are $C^{1}$ and have bounded curvature.

ACM Transactions on Graphics, Vol. V, No. N, Month 20YY. 


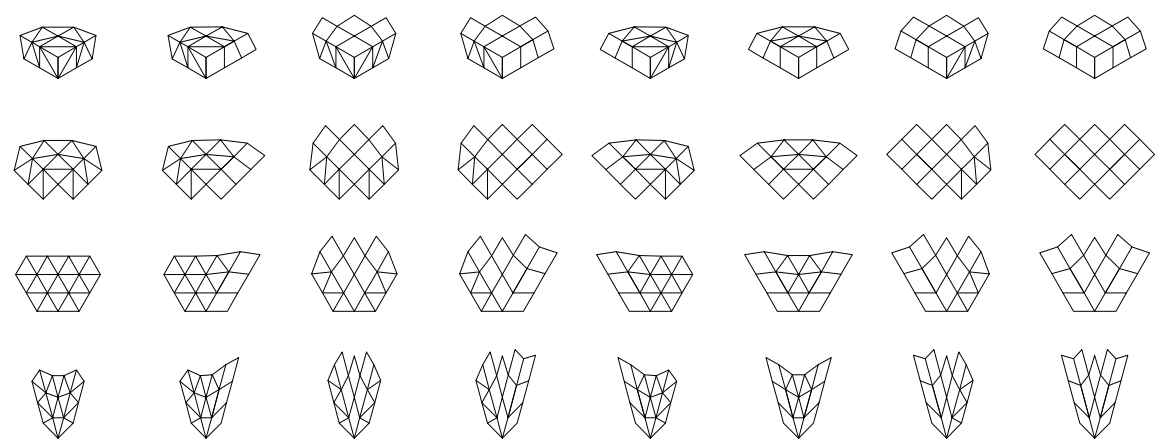

Fig. 15. Control nets of the extended sector of the characteristic map for (from top) $n=3,4,6,12$ and (left to right) the 8 possible sequences of a triangle or a quad with tri or quad neighbors. For $n=3$, the eon is an interior node. For $n=12$, it is the lowest node.

PROOF. We first show injectivity of the characteristic map. We may assume (A). Since rules (2a) and (2b) weigh both sides of a sector boundary equally, (S1) implies that $\phi_{j}$ maps the domain boundary [ $\left[\begin{array}{l}1 \\ 0\end{array}\right] . .\left[\begin{array}{l}2 \\ 0\end{array}\right]$ on a segment of the (positive) $x$-axis. Since the 4-3 transition rules are locally injective for generic data and (S2) implies that the data are generic, the image of the domain boundary does not fold back onto itself. This argument holds for all 8 configurations so that any overlap of $\phi_{i}$ with $\phi_{j}$ for $i \neq j$ is already detectable is a lack of injectivity of $\phi_{i}$. The proof therefore reduces to showing injectivity for each type of extended sector.

The characteristic map is evaluated by subdivision rules 1,2,3, but not 4 . As in [Peters and Reif 1998; Umlauf 1999], we observe that, due to the underlying box spline basis functions and rule 2, the derivatives of $\phi_{i}$ are a convex combination of the differences in the directions $d_{0}, d_{1}$ of the extended sector net. By (S3), the two families of difference vectors lie in half-cones strictly to one side of zero and by (S4) these half-cones do not overlap, i.e. they only intersect at the origin (cf. Figure 16). Therefore any vector generated as a convex combination of one family is linear independent from vectors generated by a convex combination of the vectors of the other family. Since the vectors are non-zero, $\phi$ is regular.

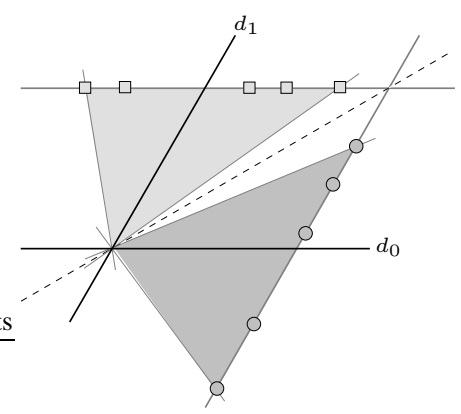

Fig. 16. (right:) Non-overlapping difference half-cones. 
By (S5) and since 4-3 transition guarantees injectivity at the endpoints of $E:=\left[\begin{array}{l}0 \\ 1\end{array}\right] . .\left[\begin{array}{l}1 \\ 0\end{array}\right]$, $\phi_{i}$ is injective along the line segment $E$. Since $\phi_{i}\left(\left[\begin{array}{l}0 \\ 2\end{array}\right] . .\left[\begin{array}{l}2 \\ 0\end{array}\right]\right)$ is a scaled image of $\phi_{i}(E)$ and since $\phi_{i}$ was shown above to be injective on the sector boundaries, $\phi_{i}$ is injective on all four boundaries. The argument of Lemma 4.1 [Peters and Reif 1998] shows that if $\phi_{i}$ is regular on $\omega$ and injective on the boundaries of $\omega$, then $\phi_{i}$ is injective on $\omega$. (The argument, in a nutshell is: assume the map is regular and injective on the boundary but not injective in the interior. Then one can trace a path of non-injective points to the boundary or the path ends in a singular point. Either conclusion contradicts the assumption.)

For $\left|\frac{1}{2}-\alpha\right| \leq \frac{1}{4}$, the eigenvalues of $\mathrm{S}$ are always positive and, in descending order, $1, \frac{1}{2}, \frac{1}{2}, \frac{1}{4}, \ldots, \frac{1}{8}, \ldots$ where the multiplicity of $\frac{1}{4}$ is $n+n_{\square}-3$.

In particular, choosing $\alpha=1 / 4$ for $n=3$ guarantees a descending sequence $1, \frac{1}{2}, \frac{1}{2}, \frac{1}{4}, \ldots$ of eigenvalues for all $n$.

To rigorously establish the convex hull property, we define an alternative rule to (4a). Let $v_{*}$ be an eon with direct neighbors $v_{j}, j=0 . . n-1$, and $w_{i}$ the fourth node of a quad $q_{i}$ with vertices $v_{*}, v_{i-1}, w_{i}, v_{i}$. Rule (4a) computes the new eon as $v_{*}^{\text {new }}:=\alpha v_{*}+\sum_{j} \beta_{j} v_{j}$. Rule (4a') computes the new eon as

$$
v_{*}^{\text {new }}:=\alpha v_{*}+\sum_{j} \beta_{j} v_{j}+\frac{1}{16 n} \sum_{q_{i}}\left(w_{i}-\frac{v_{i}+v_{i-1}}{2}\right)
$$

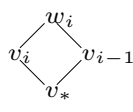

That is, for every quad facet $i$, we reduce the contribution of the two direct neighbors by one half of the weight $\frac{1}{16 n}$ given to $w_{i}$.

LEMMA 3.8 (CONVEX HULL PROPERTY). The 4-3 subdivision surface lies in the convex hull of its control points, if, for $n>3$ and subdivision steps $\sigma \leq \sigma_{0}, \frac{\sigma-1}{\sigma}$ times rule (4a') is added to $\frac{1}{\sigma}$ times rule $(4 a)$.

ProOF. All subdivision stencils in Figure 4 have entries summing to one, and they are nonnegative except for some $\beta_{i}$ in rule (4b) if $n>3$. (For $n=3$ all entries are nonnegative and we are done.) For $n>3$, since $\frac{1}{4} \leq \alpha \leq \frac{3}{4}$,

$$
\beta_{i} \geq \frac{-1}{n} \quad \text { and } \quad \beta \geq \frac{1}{4 n} .
$$

With the help of the masks and stencils of Figure 17, we can compute the contribution of a node $v_{i}$ to node $v_{0}$ after the first two subdivision steps. Here $\epsilon_{i-1}=2$, if $v_{i-1}, v_{*}, v_{i}$ form the corner of a triangle and $\epsilon_{i-1}=1$ if they form a corner of a quad. First, we show

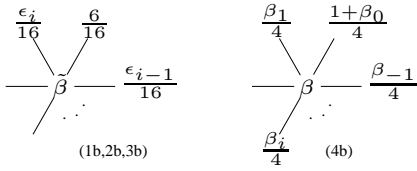

mask (contribution of $v_{i}$ )

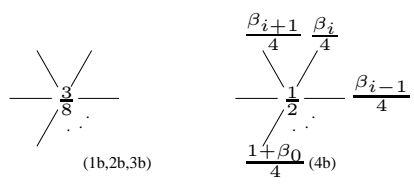

stencil (collection of $v_{0}$ )

Fig. 17. Contribution of $v_{i}$ to $v_{0}$ after two steps of subdivision. Here $\tilde{\beta}:=\beta-\frac{\gamma_{i}}{32 n}$ and some entries that will strengthen the convex hull property, say for $n=4$ and $n=5$, have been left out.

that the contribution of $v_{i}$ to $v_{0}$ after the second subdivision step, $\sigma=2$, is non-negative. 
In the first step, (4b) is not used since $(\sigma-1) / \sigma=0$. So the contribution is computed by multiplying each entry in the left mask first with the corresponding entry of the left stencil and summing the terms, then repeating the process with the right stencil and then averaging the two sums. This yields, as claimed,

$$
\begin{aligned}
\frac{1}{2} \frac{3}{8} \tilde{\beta} & +\frac{1}{2} \frac{1}{4}\left(\frac{6 \beta_{i}+\epsilon_{i-1} \beta_{i-1}+\epsilon_{i} \beta_{i+1}}{16}+2 \tilde{\beta}\right) \\
& \geq \frac{1}{16 n}\left(-1-\frac{\epsilon_{i-1}+\epsilon_{i}-2}{8}+7\left(\frac{1}{4}-\frac{\epsilon_{i-1}+\epsilon_{i}-2}{32}\right) \geq \frac{1}{16 n}\left(\frac{3}{4}-\frac{2}{32}(4+7)\right)>0 .\right.
\end{aligned}
$$

The negative summands decrease for $\sigma \leq \sigma_{0}$ and, after iteration $\sigma_{0}$, the contribution is computed by applying the two stencils exclusively to the right mask, yielding

$$
\frac{\beta}{2}+\frac{1}{16} \sum_{j} \beta_{j} \beta_{i-j}+\frac{2 \beta_{i}}{16} \geq \frac{1}{16 n}(2+1-2)>0
$$

since $\sum_{j} \beta_{j} \beta_{i-j}=\frac{1}{n}$. Rule (4a') was introduced to make the contribution of $w_{i}$ nonnegative. Nonnegativity is obvious in step 1 when only the regular rule is applied. In step 2 , the contribution is

$$
\frac{\beta_{i}+\beta_{i-1}}{64}+\frac{1}{16 n} \frac{1}{2} \geq 0 .
$$

Thus, all weights associated with the neighbors of the eon are nonnegative and sum to 1 and all rules form only convex combinations of nodes in the convex hull.

While formally necessary for the convex hull proof, the special rule (4a') is not mentioned in Section 2, since the contribution of a node $w_{i}$ to a node $v_{0}$ after two subdivision steps is bounded below by $\frac{-1}{64 n}$ and $n \geq 4$. Even a major overestimate ${ }^{5}$ that $n / 2$ nodes are weighted by the maximal negative factor, their combined contribution is only $\frac{-1}{128}$. Only a dramatically lopsided input mesh would allow this contribution to dominate the contributions of the other $n / 2$ nodes and push the surface outside the convex hull.

\section{IMPLEMENTATION AND EXAMPLES}

We implemented 4-3 subdivision to be compatible with future hardware shader support [Shiue et al. 2003], using a data structure similar to [Peters 2000; Pulli and Segal 1996]: a connectivity structure that stays fixed during the subdivision plus regular arrays for the facets whose entries roughly quadruple with each step. In this framework, it is natural to compute the new direct edge neighbors of the eons first with the regular stencils $(1 b, 2 b, 3 b)$ of Section 2 and then blend them with rule (4b). This strategy preserves features modeled by unequal distribution of neighbors of the eon and results in satisfactory curvature for low valences $n>3$, so that we do not optimize the parameter $\alpha$ as in [Stam and Loop 2003].

The example illustrate that the shapes generated by 4-3 subdivision are remarkably similar to their cousins but are superior when it comes to modeling diagonal features thanks to the switch from $\mathcal{A}_{C C}$ to $\mathcal{A}_{4}$.

Figure 19 and Figure 18 show 4-3 subdivision for simple objects with predictable shape, low valence eons, simple saddles and many transitions between quadrilateral and triangular facets.

Figure 21 compares 4-3 subdivision with Catmull-Clark, quad-tri subdivision [Stam and

${ }^{5}$ This is an overestimate since the cos terms cannot all be -1 
Loop 2003] and Loop subdivision. The point of the juxtaposition is that the rough shapes, top and bottom, are identical. That is, the superior properties of 4-3 subdivision along diagonals and at extraordinary points are not paid for by a deterioration in the overall shape. The drum-shape in Figure 20 is a challenge to schemes without geometry-dependent stencils. Only midedge subdivision [Peters and Reif 1997; Habib and Warren 1999] appears to preserve the shape along the rim while subdivision schemes with higher smoothness create an oscillating rim due to the uneven placement of the indirect neighbors of the high-valent eon. For 4-3 subdivision, this oscillation is both more pronounced and more confined compared to quad-tri subdivision, or Catmull-Clark or Loop on the suitably modified meshes. The latter export the ripples into the drum's cylinder. Figure 22, bottom illustrates how the removal of diagonals can clean up a model. Figure 23 shows how the framework facilitates displacement mapping in the spirit of [Lee et al. 2000]. Whereas in [Biermann et al. 2002] the mesh is separated and boundaries are introduced for every feature line, and there a separate suite of univariate boundary rules is applied, here a single subdivision net and surface are retained. Figure 24 illustrates the use of semi-smooth and sharp creases when modeling with 4-3 subdivision.
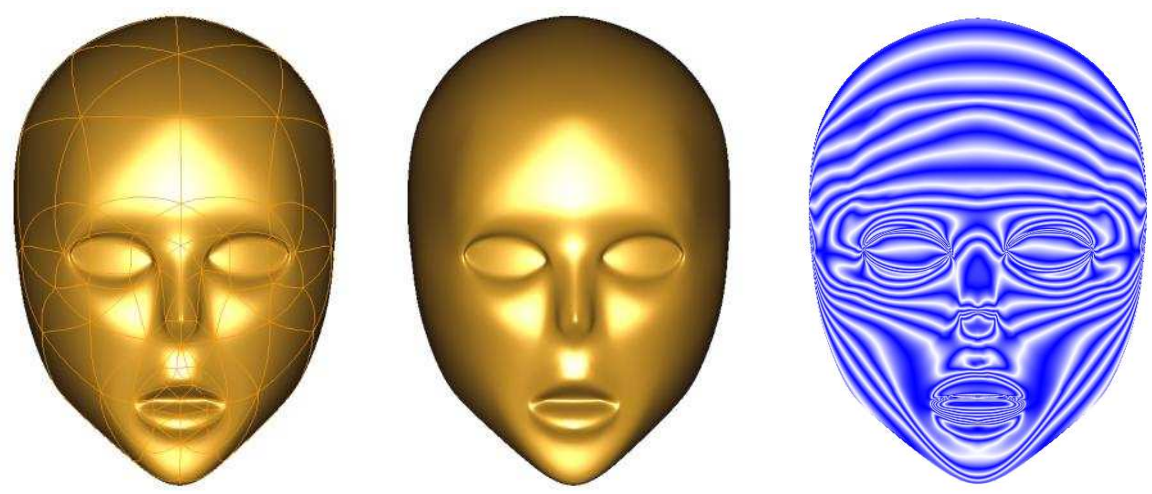

Fig. 18. Combining quads and triangles allows removing artificial diagonals. (right) High-light lines.

\section{SUMMARY}

By defining when a subdivision scheme is 'ripple-free in a direction', this paper introduced a notion of shape preservation that is at once modest (insufficient to characterize fairness of surfaces) and ambitious, in that any stronger demand for shape preservation is likely to be incompatible with a key asset of regular regions of subdivision surfaces: to be defined by a finite number of basis functions of finite support.

One can argue that the two diagonals and the coordinates axes are the only relevant griddirections to consider. Raising, say every control point at multiples of $\left[\begin{array}{l}2 \\ 1\end{array}\right]$ on a quad grid (a rook's move on a chess board) would skip enough nodes in-between to make a designer expect a ripple in the resulting surface. With the $\mathcal{A}_{4}$ subdivision rules, we have the unique, $3 \times 3$ footprint scheme that is ripple-free in all four directions and the $\mathcal{A}_{3}$ rules do an equally good job along triangle directions. 
The quad component of 4-3 subdivision has not before been used and analyzed in detail despite its small unfactored footprint and its place in the gallery of subdivision algorithms. We made an effort to analyze it, and the overall scheme, to rigorous standards.

Acknowledgements: The input mesh of Figure 18 was modified, by removing diagonals from an original contributed via ATI from id Software. We thank Georg Umlauf, Adi Levin and Hartmut Prautzsch for their constructive feedback. This research was supported by the grant NSF 9457806-CCR.

\section{REFERENCES}

Biermann, H., Levin, A., AND Zorin, D. 2000. Piecewise smooth subdivision surfaces with normal control. In Siggraph 2000, Computer Graphics Proceedings, K. Akeley, Ed. 113-120.

Biermann, H., Martin, I., Bernardini, F., AND Zorin, D. 2002. Cut-and-paste editing of multiresolution surfaces. In SIGGRAPH 2002 Conference Proceedings, J. Hughes, Ed. 312-321.

CATMull, E. AND ClaRK, J. 1978. Recursively generated B-spline surfaces on arbitrary topological meshes. Computer Aided Design 10, 350-355.

De Boor, C., Höllig, K., And Riemenschneider, S. 1993. Box splines. Applied Mathematical Sciences, vol. 98. Springer-Verlag, New York.

DeRose, T., Kass, M., And Truong, T. 1998. Subdivision surfaces in character animation. In Siggraph 1998, Computer Graphics Proceedings, M. Cohen, Ed. 85-94.

Diewald, U., Morigi, S., And RumpF, M. 2002. A cascadic geometric filtering approach to subdivision. Computer Aided Geometric Design 19, 9 (December), 675-694.

Doo, D. AND SABIN, M. 1978. Behaviour of recursive division surfaces near extraordinary points. ComputerAided Design 10, 356-360.

Habib, A. And Warren, J. 1999. Edge and vertex insertion for a class of $C^{1}$ subdivision surfaces. Comput. Aided Geom. Design 16, 4, 223-247.

Halstead, M., Kass, M., And DeRose, T. 1993. Efficient, fair interpolation using Catmull-Clark surfaces. In Siggraph 1993, Computer Graphics Proceedings, J. T. Kajiya, Ed. 35-44.

Iske, A., QuAK, E., And Floater, M., Eds. 2002. Tutorials on Multiresolution in Geometric Modeling. Springer Verlag.

Khodakovsky, A. And Schröder, P. 1999. Fine level feature editing for subdivision surfaces. In Proceedings of the Fifth Symposium on Solid Modeling and Applications (SM-99), W. F. Bronsvoort and D. C. Anderson, Eds. ACM Press, New York, 203-211.

KobBelt, L. 1996. A variational approach to subdivision. Computer Aided Geometric Design, 743-761.

Kobbelt, L. 2000. sqrt(3) subdivision. In Siggraph 2000, Computer Graphics Proceedings, K. Akeley, Ed. $103-112$.

KobBelt, L. AND SCHRÖDER, P. 1998. A multiresolution framework for variational subdivision. ACM Transactions on Graphics 17, 4 (Oct.), 209-237.

Lee, A., Moreton, H., And Hoppe, H. 2000. Displaced subdivision surfaces. In Siggraph 2000, Computer Graphics Proceedings, K. Akeley, Ed. 85-94.

LEvin, A. 2003. Polynomial generation and quasi-interpolation in stationary non-uniform subdivision. ComputerAided Geometric Design 20, 41-60.

Levin, A. ANd Levin, D. 2003. Analysis of quasi-uniform subdivision. Appl Comput Harmon Anal 15, 18-32.

Loop, C. T. 1987. Smooth subdivision surfaces based on triangles. Master's Thesis, Department of Mathematics, University of Utah.

Peters, J. 2000. Patching Catmull-Clark meshes. In Siggraph 2000, Computer Graphics Proceedings, K. Akeley, Ed. 255-258.

PETERS, J. AND REIF, U. 1997. The simplest subdivision scheme for smoothing polyhedra. ACM Transactions on Graphics 16, 4 (Oct.), 420-431.

Peters, J. AND REIF, U. 1998. Analysis of generalized B-spline subdivision algorithms. SIAM J. on Numer. Anal. 35, 2 (Apr.), 728-748.

Peters, J. AND REIF, U. 2004. Shape characterization of subdivision surfaces - basic principles. CAGD. to appear. 
Prautzsch, H. And Boehm, W. 2002. Handbook of Computer Aided Geometric Design. North-Holland, New York, Chapter 10 Box splines, 255-283.

Prautzsch, H. and Umlauf, G. 1998. Improved triangular subdivision schemes. In Proceedings of the Conference on Computer Graphics International 1998 (CGI-98), F.-E. Wolter and N. M. Patrikalakis, Eds. IEEE Computer Society, Los Alamitos, California, 626-632.

Pulli, K. And Segal, M. 1996. Fast rendering of subdivision surfaces. In Proceedings of the eurographics workshop on Rendering techniques '96. Springer-Verlag, 61-70.

REIF, U. 1995. A unified approach to subdivision algorithms near extraordinary vertices. Computer Aided Geometric Design 12, 2, 153-174.

REIF, U. 1999. Analyse und Konstruktion von Subdivisionsalgorithmen für Freiformflächen beliebiger Topologie. Shaker Verlag, Aachen.

Rioul, O. 1992. Simple regularity criteria for subdivision schemes. SIAM J. Math. Anal. 23, 6, 1544-1576.

Ron, A. AND SHEN, Z. 1998. Compactly supported tight affine spline frames in $L_{2}\left(\mathbf{R}^{d}\right)$. Math. Comp. 67, 221, 191-207.

Sabin, M. 1991. Cubic recursive division with bounded curvature. Curves and Surfaces, 411-414.

Shiue, L.-J., Goel, V., And Peters, J. 2003. Mesh mutation in programmable graphics hardware. In Proceedings of the ACM SIGGRAPH/EUROGRAPHICS conference on Graphics hardware. Eurographics Association, $15-24$.

STAM, J. 2001. On subdivision schemes generalizing uniform $B$-spline surfaces of arbitrary degree. Comput. Aided Geom. Design 18, 5, 383-396.

STAM, J. AND Loop, C. 2003. Quad/triangle subdivision. Computer Graphics Forum 22, 1, 1-7.

UMLAUF, G. 1999. Glatte Freiformflächen und optimierte Unterteilungsalgorithmen. Shaker Verlag, Aachen. Velho, L. And Zorin, D. 2001. 4-8 subdivision. Computer Aided Geometric Design 18, 5 (June), 397-427. Warren, J. AND Weimer, H. 2002. Subdivision Methods for Geometric Design. Morgan Kaufmann Publishers, New York.

ZORIN, D. AND SCHRÖDER, P. 2001. A unified framework for primal/dual quadrilateral subdivision schemes. Comput. Aided Geom. Design 18, 5, 429-454.
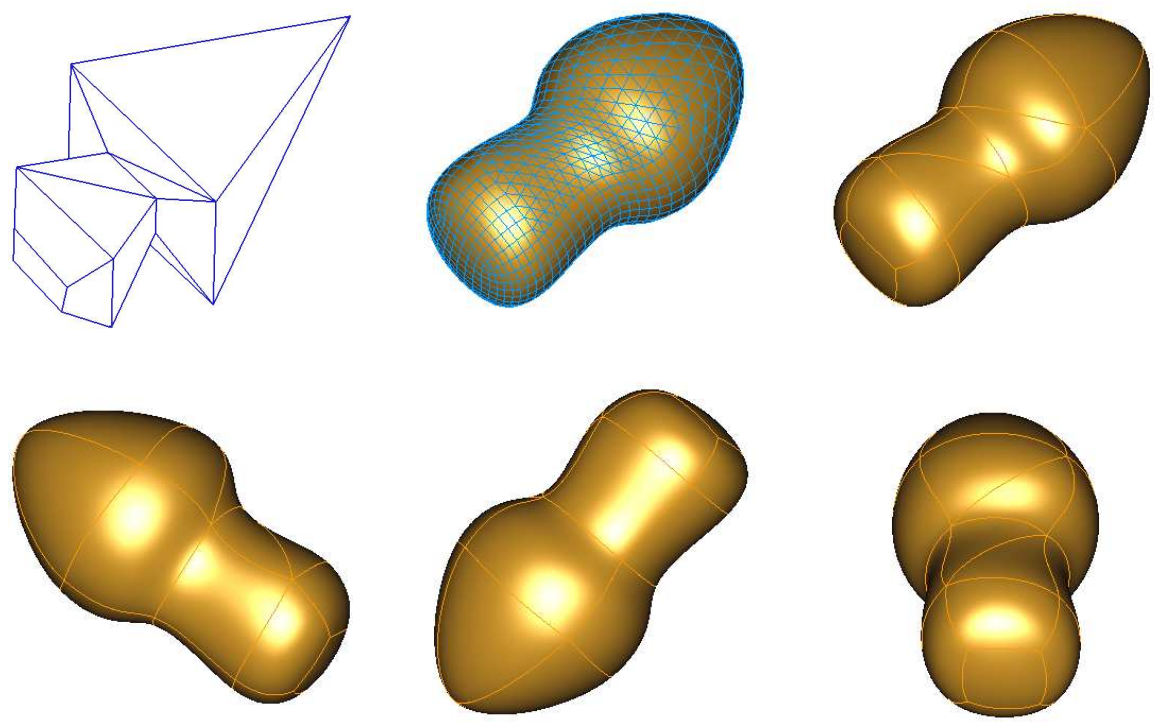

Fig. 19. 4-3 subdivision: simple shape with eons of low valence and quad to triangle transitions. 


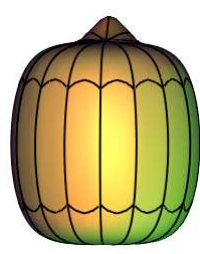

Quad-tri

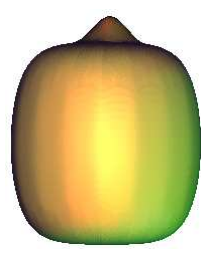

Quad-tri

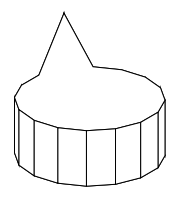

Input Mesh

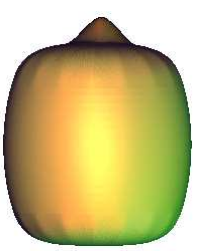

4-3

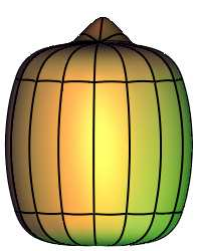

$4-3$

Fig. 20. High valence and low valence eons. The eon at the flat bottom of the drum-shape is the center of 16 triangle facets. This high-valent eon and its 4-valent neighbors cause ripples. (left two) Quad-tri subdivision spreads these ripples to the faces of the drum shape. This is made visible by the wavy boundary between quad and triangle regions. (right two) The same boundary is much straighter for 4-3 subdivision. The quad region is insulated from the ripples at the price of more pronounced oscillations in the triangle region.
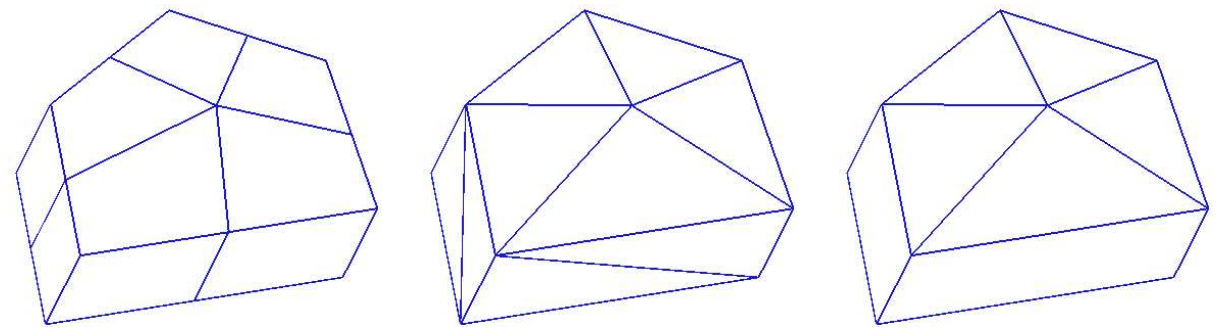

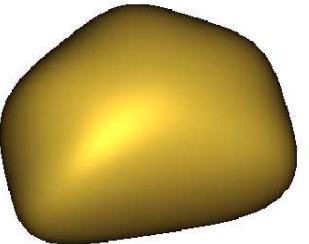

$\mathrm{CC}$

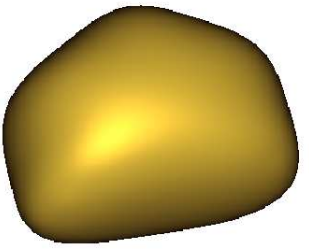

4-3

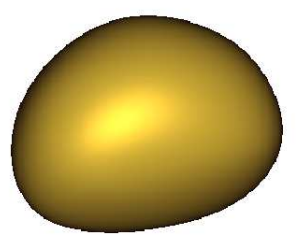

Loop

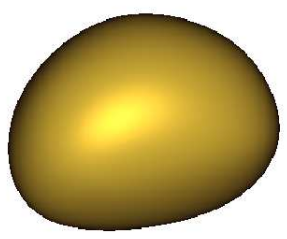

4-3

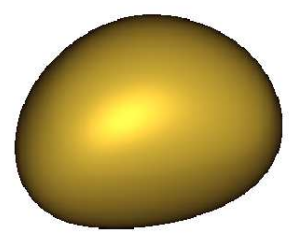

Quad-Tri

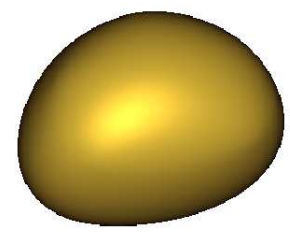

4-3

Fig. 21. Comparison of 4-3 Subdivision with Catmull-Clark (CC), Loop and Quad-Tri subdivision. 

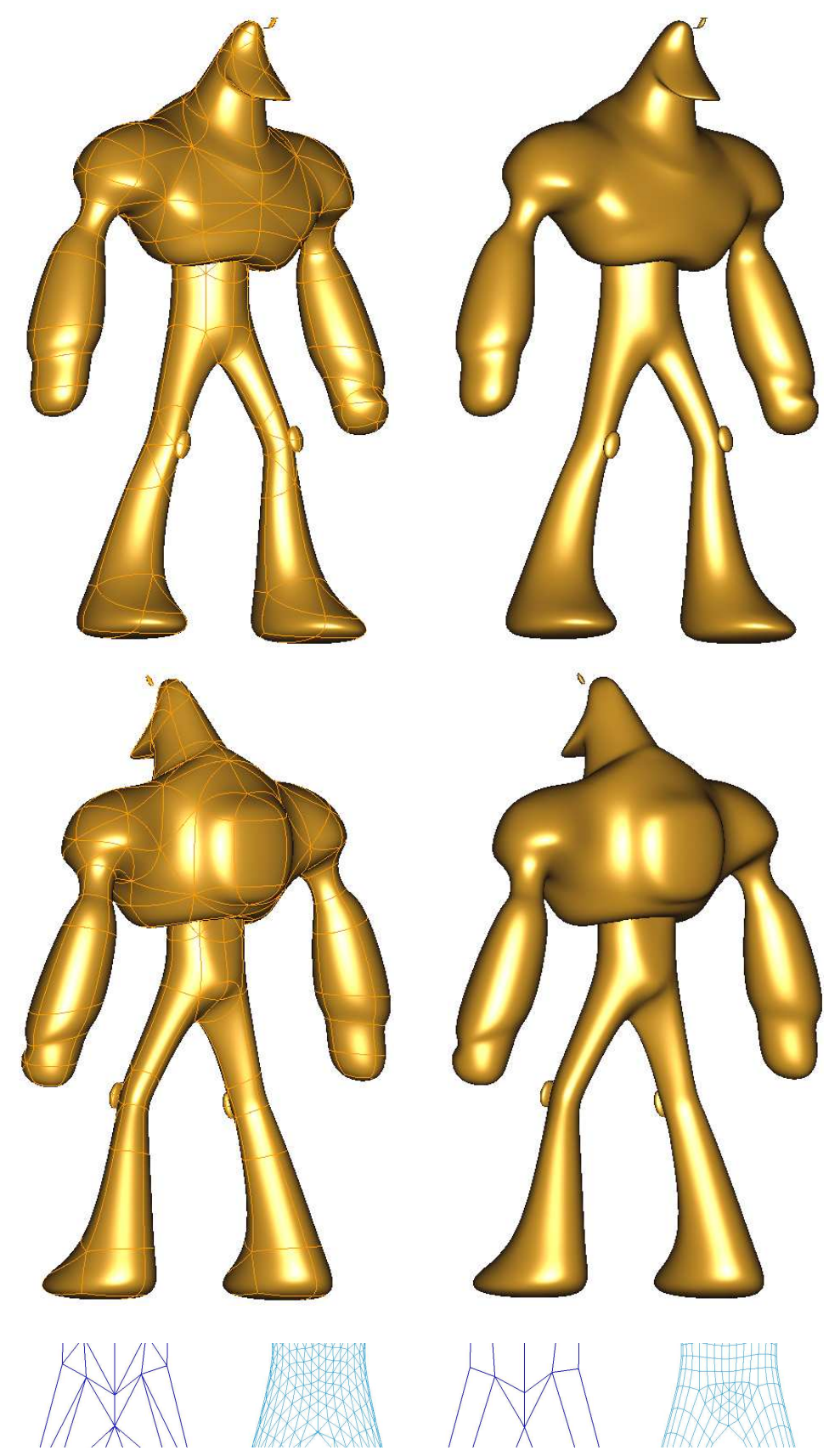

Fig. 22. (top and middle) 4-3 subdivision of a complex model. On the left, the images of the original facet boundaries are drawn to indicate the input quads and triangles. On the right, these lines are removed to fully uncover the surface. (bottom) Removal of artificial diagonals. 

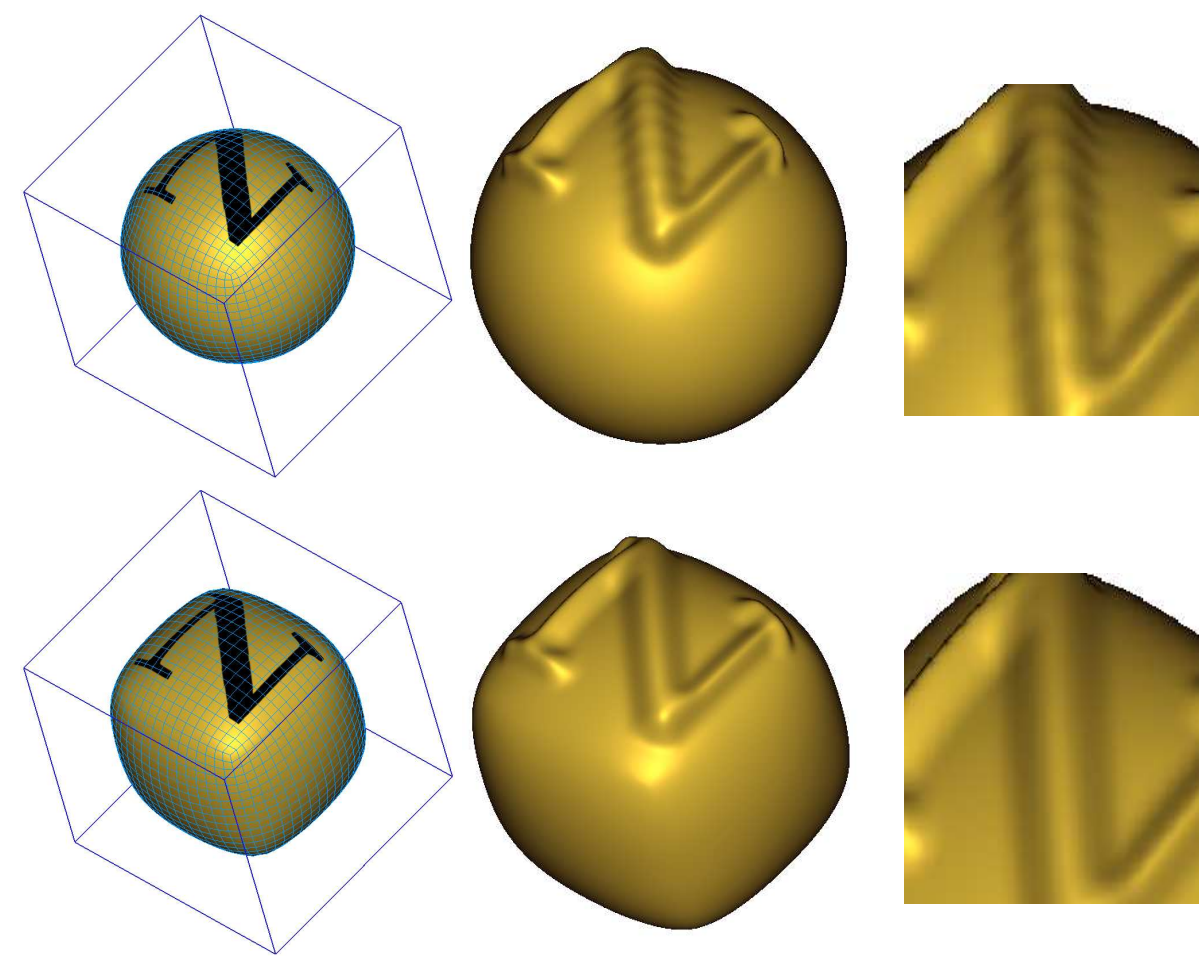

Fig. 23. Texture-controlled displacement after three steps of subdivision. (top) Catmull-Clark subdivision, displacement, plus two additional Catmull-Clark subdivision steps. (bottom) 4-3 subdivision using $\alpha=0.6$, displacement, smoothed by two additional 4-3 subdivision steps.
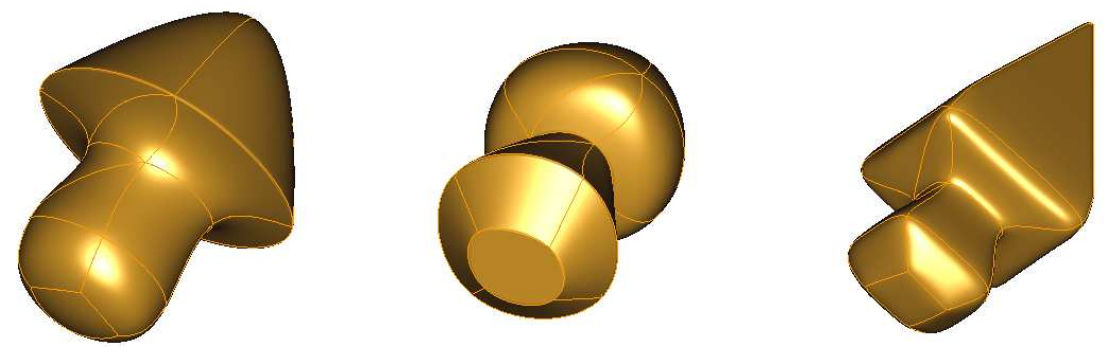

Fig. 24. Creases and semi-smooth creases added to the data of Figure 19.

Received Month Year; revised Month Year; accepted Month Year 